\title{
Clinical recommendations for
} cardiovascular magnetic resonance mapping of $\mathrm{T} 1, \mathrm{~T} 2, \mathrm{~T}_{2}^{*}$ and extracellular volume: A consensus statement by the Society for Cardiovascular Magnetic Resonance (SCMR) endorsed by the European Association for Cardiovascular Imaging (EACVI)

Daniel R. Messroghli, ${ }^{1,2,3^{*}}$, James C. Moon ${ }^{4}$, Vanessa M. Ferreira ${ }^{5}$, Lars Grosse-Wortmann ${ }^{6}$, Taigang He ${ }^{7}$, Peter Kellman ${ }^{8}$, Julia Mascherbauer ${ }^{9}$, Reza Nezafat ${ }^{10}$, Michael Salerno ${ }^{11}$, Erik B. Schelbert ${ }^{12,13,14}$, Andrew J. Taylor ${ }^{15}$, Richard Thompson ${ }^{16}$, Martin Ugander ${ }^{17}$, Ruud B. van Heeswijk ${ }^{18}$ and Matthias G. Friedrich ${ }^{19,20,21,22}$

\begin{abstract}
Parametric mapping techniques provide a non-invasive tool for quantifying tissue alterations in myocardial disease in those eligible for cardiovascular magnetic resonance (CMR). Parametric mapping with CMR now permits the routine spatial visualization and quantification of changes in myocardial composition based on changes in T1, T2, and T2*(star) relaxation times and extracellular volume (ECV). These changes include specific disease pathways related to mainly intracellular disturbances of the cardiomyocyte (e.g., iron overload, or glycosphingolipid accumulation in Anderson-Fabry disease); extracellular disturbances in the myocardial interstitium (e.g., myocardial fibrosis or cardiac amyloidosis from accumulation of collagen or amyloid proteins, respectively); or both (myocardial edema with increased intracellular and/or extracellular water). Parametric mapping promises improvements in patient care through advances in quantitative diagnostics, inter- and intra-patient comparability, and relatedly improvements in treatment. There is a multitude of technical approaches and potential applications. This document provides a summary of the existing evidence for the clinical value of parametric mapping in the heart as of mid 2017, and gives recommendations for practical use in different clinical scenarios for scientists, clinicians, and CMR manufacturers.
\end{abstract}

\footnotetext{
* Correspondence: dmessroghli@dhzb.de

'Department of Internal Medicine and Cardiology, Deutsches Herzzentrum Berlin, Berlin, Germany

${ }^{2}$ Department of Internal Medicine and Cardiology, Charité

Universitätsmedizin Berlin, Augustenburger Platz 1, 13353 Berlin, Germany

Full list of author information is available at the end of the article
} 


\section{Background}

CMR is the primary imaging modality for myocardial tissue characterization. CMR parametric mapping now permits the routine spatial visualization of quantitative changes in myocardium based on changes in myocardial parameters T1, T2, T2*(star) and ECV. These changes include specific disease pathways related to mainly intracellular disturbances of the cardiomyocyte (e.g., iron overload, or glycosphingolipid accumulation in Anderson-Fabry disease); extracellular disturbances in the myocardial interstitium (e.g., myocardial fibrosis of cardiac amyloidosis from accumulation of collagen or amyloid proteins, respectively); or both (e.g. myocardial edema and/or infarction with increased intracellular and/or extracellular water). Unlike T1-, T2-, or T2*-weighted images, mapping permits both visualization and quantification of the disease process, independent of whether myocardial disease is focal or diffuse. This innovation is important because historically, diffuse myocardial disease related to specific disease pathways has been difficult to non-invasively quantify or even appreciate.

Our technical capabilities with parametric mapping may exceed our understanding of how this data can guide optimal treatment for patients with signs or symptoms of underlying disease. Nonetheless, there is an important precedent demonstrating that image guided care exploiting CMR parametric measurements can improve patient outcome in iron overload states [1]. Thus, advances in CMR parametric mapping promise to improve patient care though better diagnostic decisionmaking, which in turn can result in better treatment, as a major step towards Precision Medicine. In addition, CMR parametric mapping also promises to facilitate the development of novel therapeutics, by providing quantitative endpoints reflective of the disease pathway of interest, especially in phase 2 efficacy trials. Technological advances now permit routine acquisition of parametric maps in patients eligible for CMR. While mapping adds unique and relevant diagnostic information on the status of the myocardium, its clinical application requires specific hardware, software, data acquisition and evaluation procedures, which are not completely standardized.

\section{Aims and scope of this document}

This document provides recommendations for clinical and research applications of CMR myocardial T1, T2, $\mathrm{T} 2 *$, and ECV mapping. We cite published evidence when available and provide expert consensus where incomplete. We recognize a priori that multiple methodologies for CMR parametric mapping do and should exist, with continued evolution and residual imperfections. Despite these limitations, abundant evidence demonstrates that parametric mapping appears robust under many conditions in its present form. We make analogy to another key cardiac imaging biomarker, the left ventricular ejection fraction (LVEF), where measurement variations persist within and across modalities, yet the yield of biological information is sufficient to diagnose disease, guide and monitor treatment, and to predict outcome. CMR parametric mapping goes beyond nonspecific functional surrogate markers of cardiovascular disease such as LVEF. Rather, CMR parametric mapping offers the potential to examine specific disease pathways that affect myocardial tissue composition.

In 2013, the "T1 Mapping Development Group" published a consensus statement that proposed suitable terminology and specific recommendations for site preparation, scan types, scan planning and acquisition, quality control, visualization and analysis, and technical directions [2]. Building on this initiative, the Consensus Group on Cardiac MR Mapping has formed itself to provide guidance on CMR mapping to scientists, clinicians, and manufacturers. The team includes experts with a wide and representative range of technical and clinical expertise, a broad geographical base and a balanced spectrum of interest.

Considering the rapidly increasing interest in mappingbased myocardial tissue characterization, the group developed this document to provide 1) an update on the available experimental and clinical evidence, 2) an updated list of clinical indications, 3) practical recommendations for stateof-the-art protocols and techniques, and 4) guidance for research.

\section{Terminology}

Table 1 provides definitions for terminology related to the field of parametric mapping of the heart. We now recommend that ECV be expressed as a percentage (e.g. $25 \%$ rather than 0.25 ).

\section{Recommendations part I: Clinical indications and utility}

1 Parametric mapping is useful in patients undergoing evaluation for suspected myocardial disease, and masses.

2 In the clinical scenarios of potential iron overload, amyloidosis, Anderson-Fabry disease, and myocarditis, cardiac mapping provides unique information to guide clinical care and should be applied (Tables 2 and 3; Fig. 1).

3 Parametric mapping should be considered in the diagnostic evaluation of all patients with heart failure and unexplained troponin elevation.

4 The choice of CMR mapping techniques and protocols should be guided by the clinical context.

5 In patients receiving extracellular gadolinium-based contrast agents, routine assessment of ECV may be reasonable. 
Table 1 Definitions of technical terms in the field of parametric mapping of the heart

\begin{tabular}{|c|c|}
\hline Term & Meaning \\
\hline $\mathrm{T} 1[\mathrm{~ms}]$ & Time constant representing the recovery of longitudinal magnetization (spin-lattice relaxation) \\
\hline Native T1 & $\mathrm{T} 1$ in the absence of an exogenous contrast agent \\
\hline $\mathrm{T} 2$ [ms] & Time constant representing the decay of transverse magnetization (spin-spin relaxation) \\
\hline $\mathrm{T}^{*}[\mathrm{~ms}]$ & $\begin{array}{l}\text { Time constant representing the decay of transverse magnetization in the presence of local field } \\
\text { inhomogeneities }\end{array}$ \\
\hline ECV [\%] & 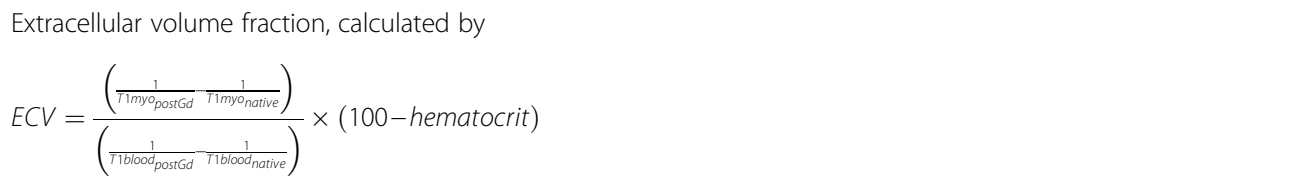 \\
\hline & where myo = myocardium; blood = intracavitary blood pool; hematocrit = cellular volume fraction of blood [\%] \\
\hline Synthetic ECV [\%] & ECV where hematocrit is not measured by laboratory blood sampling but derived from blood T1 \\
\hline Parametric mapping & $\begin{array}{l}\text { A process where a secondary image is generated in which each pixel represents a specific magnetic tissue property } \\
\left(\mathrm{T} 1, \mathrm{~T} 2 \text {, or } \mathrm{T} 2^{*}\right) \text { or a derivative such as ECV ) derived from the spatially corresponding voxel of a set of co-registered } \\
\text { magnetic resonance source images }\end{array}$ \\
\hline
\end{tabular}

Centers undertaking parametric mapping should fulfill the site requirements described in Rationale and diagnostic potential of parametric mapping techniques.

\section{Recommendations part II: Implementation Site preparation and normal values CMR systems}

1) T1, ECV and T2 mapping are typically performed at 1.5 or 3 Tesla $(\mathrm{T})$.

2) $\mathrm{T}^{*}$ mapping for iron overload currently should be performed at $1.5 \mathrm{~T}$.

\section{Pulse sequence schemes}

1) CMR mapping sequences for clinical use should have a published clinical evidence base.

Table 2 Clinical utility of parametric mapping techniques ordered by pathophysiologic mechanism and tissue characteristics. $++=$ useful; $+=$ potentially useful; ? = unknown; $-=$ not useful. *: Diffuse/global refers to findings affecting the majority of the myocardium, whereas focal/regional refers to localized, including patchy abnormalities

\begin{tabular}{lllllll}
\hline & & T1 (native) & ECV & T2 & T2* \\
\hline Infiltration & Iron & + & & $?$ & + & ++ \\
& Amyloid & ++ & & ++ & $?$ & - \\
& Anderson-Fabry & ++ & & - & + & - \\
Acute myocardial injury & Edema & ++ & + & ++ & $?$ \\
& Necrosis & ++ & & ++ & + & ++ \\
Fibrosis & Hemorrhage & + & & $?$ & + & ++ \\
& Diffuse/global* & + & & ++ & $?$ & - \\
& Focal/regional* & + & & ++ & - & - \\
\hline
\end{tabular}

2) Where mapping is clinically reported, the use of a commercial pulse sequence is preferable if supplied by the manufacturer. Other pulse sequences can be considered, but both need to fulfill (1) above.

\section{Normal/reference ranges}

1) For native $T 1$ and $T 2$ mapping, local results should be benchmarked against published reported ranges, but a local reference range should be primarily used.

2) Reference ranges should be generated from data sets that were acquired, processed, and analyzed in the same way as the intended application, with the upper and lower range of normal defined by the mean plus and minus 2 standard deviations of the normal data, respectively.

Table 3 Clinical utility of parametric mapping techniques according to expert opinion

\begin{tabular}{ll}
\hline Proven clinical utility & Iron deposition \\
& Amyloid disease \\
& Anderson-Fabry disease \\
& Myocarditis \\
\hline Potential clinical utility & Cardiomyopathy \\
& Heart failure \\
& Congenital heart disease \\
& Acute/chronic myocardial infarction \\
& Myocardial ischemia \\
& Suspected transplant rejection \\
Athlete's heart & (Para-)cardiac masses
\end{tabular}




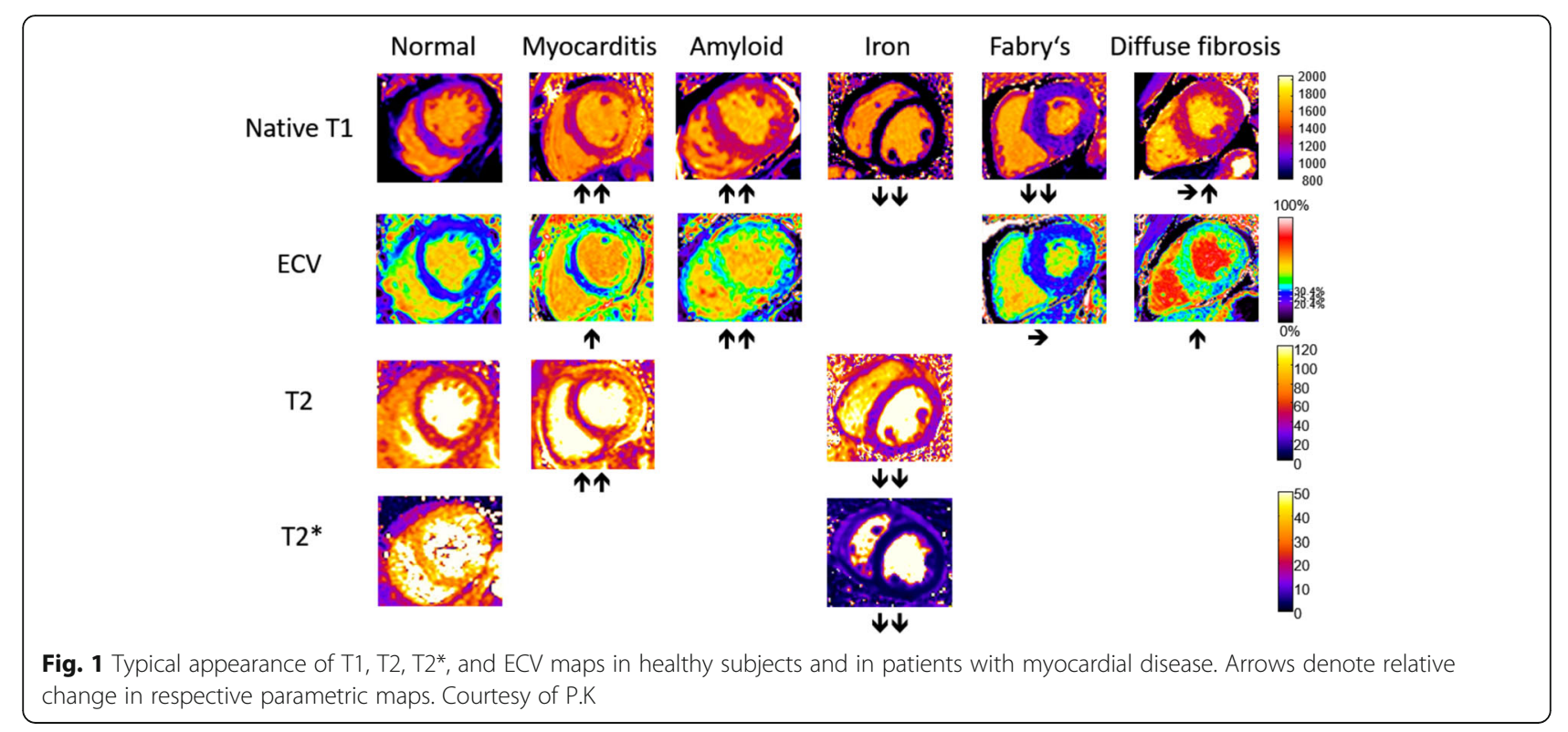

3) If local reference ranges are not available for native T1 and T2 mapping, quantitative results should not be reported clinically.

4) The required precision of the local reference range depends on the proposed clinical application:

a. For scenarios with large-magnitude biological changes (e.g. T1 for establishing diagnosis in amyloid, iron, Anderson-Fabry disease, and acute myocardial injury), lower precision is acceptable; e.g. native $\mathrm{T} 1$ and $\mathrm{T} 2$ reference ranges on the basis of 15 healthy subjects or 20 normal individuals (e.g. referred for CMR without any abnormal findings) may be sufficient.

b. For small-magnitude biological changes (e.g. diffuse myocardial fibrosis), high precision is required for native T1 and T2 mapping; e.g. gender $+/$ - age adjusted reference ranges derived from $50+$ healthy subjects.

c. For ECV, reference ranges from the literature using the same CMR system and same pulse sequence may be acceptable, as the dependence of ECV on field strength, sequence choice and imaging parameters appears lower than for native $\mathrm{T} 1$.

d. For T2*, a 3-tier risk model (low risk, >20 ms; intermediate risk, 10-20 ms; and high risk, $<10 \mathrm{~ms}$ ) for cardiac iron overload should be used if images are acquired at $1.5 \mathrm{~T}$ with $\geq 8$-point gradient echo pulse sequences.

e. Tracking changes over time requires the use of identical imaging parameters or high-precision reference ranges (see b).

\section{CMR system-related changes of normal/reference ranges} over time

1) Once a reference range is established, the major scan parameters (slice thickness, flip angles etc.), contrast agent/dose and systolic/diastolic phase should not be changed.

2) Regularly repeated phantom-based quality control is recommended to ensure that status and stability of the CMR system have not changed significantly during the time between establishing normative values and clinical scanning.

3) Phantom-based quality control should be performed every time there is a change to the CMR system (hardware, software), a software installation, and every 3 months.

\section{Imaging protocols \\ General recommendations:}

1) Native $T 1, T 2$, and $T 2 *$ are measured in the absence of contrast agents (at least $24 \mathrm{~h}$ from the last dose, if any, in patients with normal renal function).

2) Motivation and detailed instructions of patients are important to avoid incomplete breath-holds or motion artifacts.

3) In-plane motion correction is recommended if available, but is not a replacement for breathholding in non-navigated techniques.

4) Volume-selective B0 shimming focused on the heart is highly recommended at $1.5 \mathrm{~T}$, and essential at $3 \mathrm{~T}$. B1 (radiofrequency) volume shimming is recommended at $3 \mathrm{~T}$. 
5) In-plane resolution should not be increased to levels where the resulting acquisition duration of the source images exceeds the time frame within the cardiac cycle where data can be acquired without blurring effects by the giving technique, with consideration of the subject's heart rate.

6) Diastolic image acquisition is recommended if there is a regular heart rhythm.

7) In patients with tachycardia, specific sequences designed for higher heart rates can be useful.

8) In patients with atrial fibrillation, image acquisition should be repeated to allow for averaging of the results. Systolic readout has been shown to produce robust T1 maps in tachyarrhythmias but requires specific normal values.

9) In patients with pacemakers or implanted cardiodefibillators (ICDs), CMR parametric mapping is not reliable unless specific shimming algorithms or sequences can be used to minimize the impact of artifacts.

\section{T1 mapping/ECV}

1) Optimized acquisition schemes for post-contrast acquisitions can be used to gain precision.

2) For Look-Locker-based techniques (e.g. MOLLI), correction for readout-induced deflection of $\mathrm{T} 1$ relaxation is required (Look-Locker correction).

3) An extracellular contrast agent with non-protein bound distribution should be used for the assessment of ECV.

4) Gadolinium based contrast doses of $0.1-0.2 \mathrm{mmol} / \mathrm{kg}$ are recommended.

5) Hematocrit for the calculation of ECV should be obtained immediately before the scan if possible, otherwise within $24 \mathrm{~h}$ of scanning.

6) For ECV measurements, post-contrast T1 mapping should be performed $10-30$ min post contrast administration.

7) Split dose protocols (e.g. in adenosine perfusion scans) can be used to assess ECV. The timing should be taken from the last dose.

\section{T2 mapping}

1) T2-prepared balanced steady-state free precession (bSSFP) or gradient echo pulse sequences with a minimum of 3 source images are recommended.

2) GraSE or turbo spin echo (TSE) approaches may be appropriate if published data on accuracy and precision are available and favorable.

3) Two-parameter fitting is appropriate.

4) Saturation pulse acquisition may help to remove T1 recovery bias.

\section{T2* mapping}

1) If available, $\mathrm{T} 2 *$ mapping should be performed at $1.5 \mathrm{~T}$ (see also Rationale and diagnostic potential of parametric mapping techniques).

2) Multi-echo gradient echo with 8 equally spaced echoes ranging from 2 to $18 \mathrm{~ms}$ may be used at $1.5 \mathrm{~T}$.

3) A dark-blood approach is recommended if available.

4) $\mathrm{T}^{*}$ for the assessment of iron overload an interventricular septal region-of-interest (ROI) is recommended

5) For the concomitant assessment of liver T2*, the use of fat saturation and the shortest echo time (TE) available are recommended.

\section{Scan planning and acquisition}

1) Added pulse sequences should not compromise the primary study indications.

2) Proper adjustment of main magnetic field shim and center frequency should be assured to minimize off resonance.

3) Through-plane partial volume effects should be minimized where possible by choosing slice orientations that are located orthogonal to the target structures. Caution is required in short axis views of the apex.

4) Image quality should be reviewed during acquisition (e.g. by monitoring sequence sounds and electrocardiographic (ECG) gating), and by looking at source images, error maps, and other quality control maps). Scans should be repeated if suboptimal or non-diagnostic.

5) Native and post-contrast T1 maps should be acquired using the same slice prescription and other scan parameters at the same cardiac phase (but T1 sampling scheme may be changed - see also T1 mapping/ ECV).

6) A comprehensive imaging protocol for myocardial tissue characterization including parametric mapping is presented in Fig. 2. Disease-specific recommended slices and approaches are given in Table 4. We give the following recommendations: a) For global/diffuse disease, a basal and mid short axis map should be acquired with an optional single long axis map.

b) For patchy disease, the acquisition of at least one long axis map is mandatory (4-chamber for amyloid to visualize base-to-apex gradient, 3-chamber for Anderson-Fabry disease to assess basal inferolateral scar) in addition to basal and mid short axis maps. 


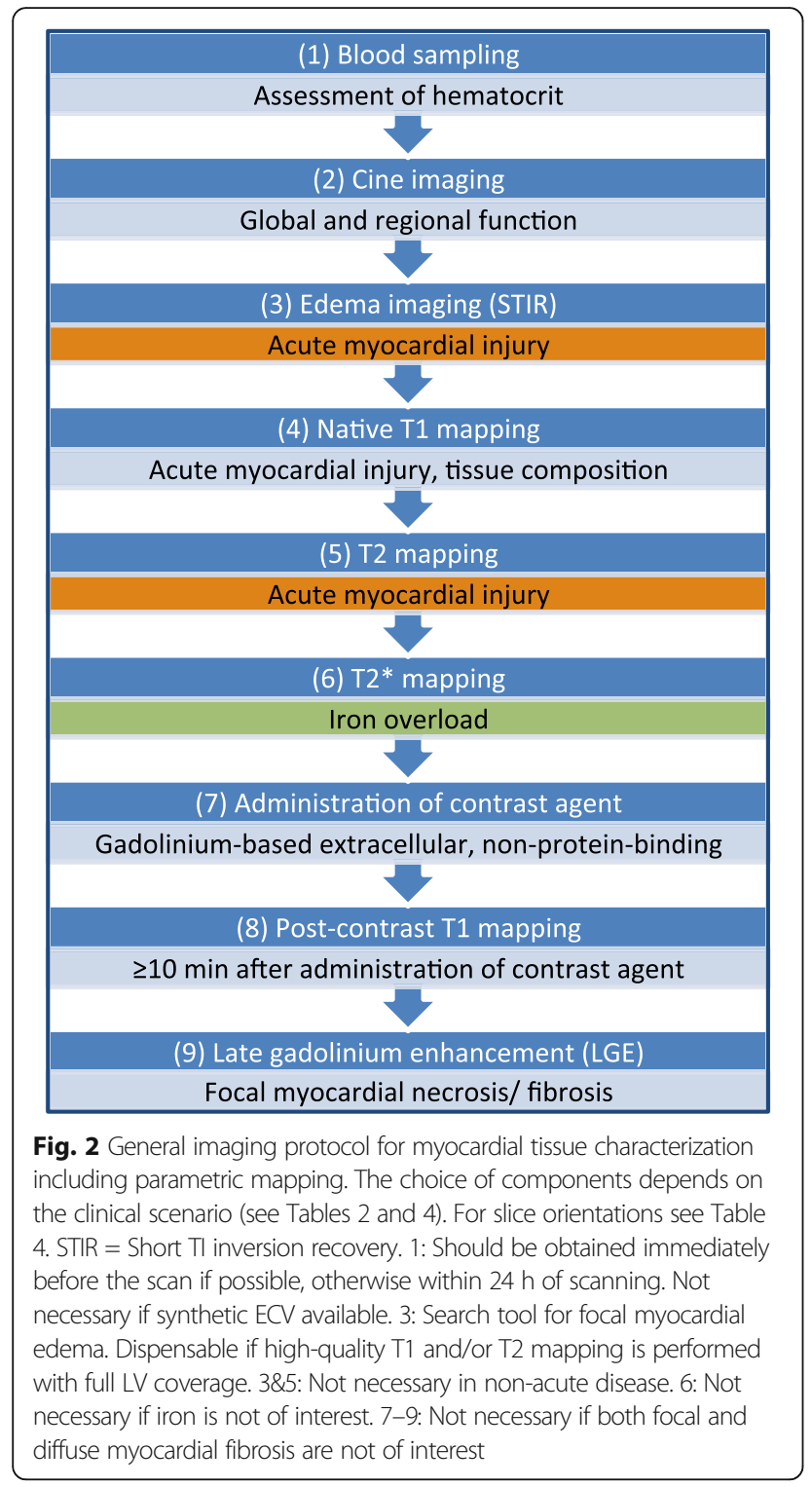

c) For focal and/or acute disease, additional short axis maps should be added and should cover an area of maximal abnormality and an area of apparently minimal abnormality (as determined on cine and/or T2-weighted images) or the whole left ventricle (if information from cine and T2weighted images should not be available at the time of image acquisition).

d) Whole heart coverage may add diagnostic yield but risks disproportionately long imaging protocols and patient fatigue.

7) Inline ECV maps (including synthetic, if the relationship between native blood T1 and hematocrit is known for the pulse sequence and field strength) can be a useful alternative to manual ECV calculations.
Table 4 Recipe table for specific parametric mapping protocols. $\mathrm{SAX}=$ short axis slice, $3 \mathrm{Ch}=3$ chamber view, $4 \mathrm{Ch}=4$ chamber view, $\mathrm{T} 1=\mathrm{T} 1$ mapping, $\mathrm{T} 2=\mathrm{T} 2$ mapping, $\mathrm{T}^{*}=\mathrm{T} 2^{*}$ mapping

\begin{tabular}{|c|c|c|}
\hline Scenario & $\begin{array}{l}\text { Pulse sequences/slice } \\
\text { orientations }\end{array}$ & Breathholds \\
\hline \multirow[t]{4}{*}{ Amyloid } & T1 mid and basal SAX, 4Ch & 7 \\
\hline & repeated post contrast & \\
\hline & T2 mid SAX, & \\
\hline & $\mathrm{T} 2^{*}-$ & \\
\hline \multirow[t]{4}{*}{ Anderson-Fabry } & T1 mid and basal SAX, 3Ch & $4-7$ \\
\hline & $\begin{array}{l}\text { repeated post contrast } \\
\text { (research) }\end{array}$ & \\
\hline & T2 basal SAX & \\
\hline & $\mathrm{T} 2^{*}-$ & \\
\hline \multirow[t]{4}{*}{ Iron overload } & T1 mid and basal SAX, 4Ch & 6 \\
\hline & not post contrast & \\
\hline & T2 liver single transverse & \\
\hline & $\begin{array}{l}\mathrm{T}^{*} \text { mid SAX, liver single } \\
\text { transverse }\end{array}$ & \\
\hline \multirow[t]{4}{*}{$\begin{array}{l}\text { Myocarditis, acute myocardial } \\
\text { infarction, other regional } \\
\text { disease }\end{array}$} & $\begin{array}{l}\text { T1 SAX multi-slice, long } \\
\text { axis (through region of } \\
\text { hyper-intensity on STIR or } \\
\text { regional wall motion } \\
\text { abnormality on cine) }\end{array}$ & $16-25$ \\
\hline & repeated post contrast & \\
\hline & T2 SAX multi-slice & \\
\hline & $\mathrm{T} 2^{*}-$ & \\
\hline \multirow[t]{4}{*}{ Diffuse fibrosis } & $\begin{array}{l}\text { T1 mid and basal SAX, 4Ch } \\
\text { (research) }\end{array}$ & 6 \\
\hline & repeated post contrast & \\
\hline & T2 (research) & \\
\hline & $\mathrm{T} 2^{*}-$ & \\
\hline
\end{tabular}

\section{Visualization and analysis}

1) Reporting clinicians should learn how to review source images and quality control maps to ensure registration/significant artifacts not present.

2) Maps may be displayed in color if the color look up tables are set according to site-specific ranges of normal, or in gray scale in combination with appropriate image processing, to highlight areas of abnormality.

3) For global assessment and diffuse disease, a single ROI should be drawn in the septum on mid-cavity short-axis maps to avoid lung, liver and veins as sources of susceptibility artifacts.

4) In case of artifacts or non-conclusive results on midcavity ROIs, basal ROIs can be used for validation.

5) For focal disease, additional ROIs might be drawn in areas of abnormal appearance on visual inspection. Very small ROIs ( $<20$ pixels) should be avoided. 


\section{T1 Mapping and ECV in clinical practice}

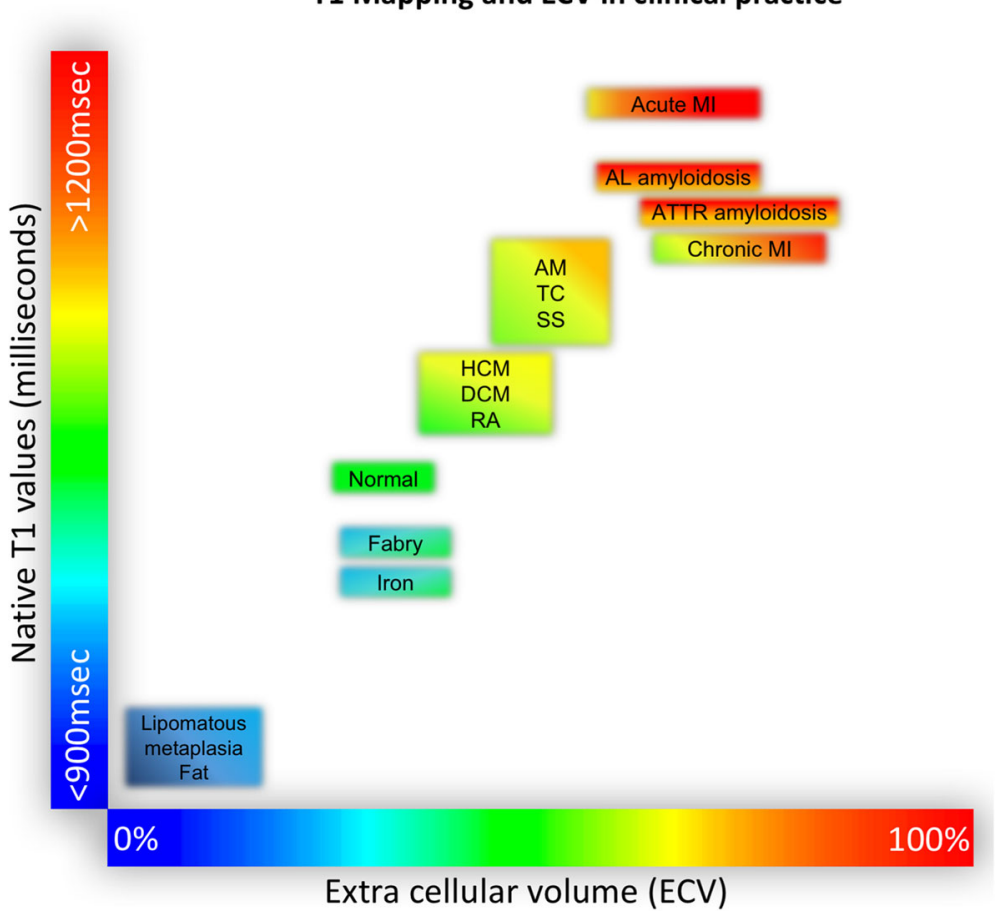

Fig. 3 Alterations of $\mathrm{T} 1$ and ECV in different myocardial diseases (reproduced with permission from [193]). T1 values refer to MOLLI-based techniques at $1.5 \mathrm{~T}$

6) ROIs should be checked if generated automatically.

7) Drawing ROIs on greyscale may avoid bias.

8) Myocardial ROIs should be placed accurately to minimize partial volume effects from adjacent blood pool or extra-myocardial tissues.

9) ROIs should be drawn independently of late gadolinium enhancement (LGE) fibrosis imaging. It is acceptable for ROIs to exclude infarcts (i.e., include remote myocardium) and include nonischemic LGE.

Table 5 Typical alterations of $\mathrm{T} 1, \mathrm{~T} 2, \mathrm{~T} 2 *$ relaxation times and ECV according to pathology. For further details see Captur et al. [194]

\begin{tabular}{llll}
\hline Measure & Decrease & Mild increase & $\begin{array}{l}\text { Moderate or } \\
\text { severe increase }\end{array}$ \\
\hline Native T1 & $\begin{array}{l}\text { Anderson-Fabry, iron } \\
\text { overload, fat, } \\
\text { hemorrhage (athlete's } \\
\text { heart) } \\
\text { athlete's heart }\end{array}$ & $\begin{array}{l}\text { diffuse fibrosis, } \\
\text { scar, subacute } \\
\text { inflammation }\end{array}$ & $\begin{array}{l}\text { amyloid, acute } \\
\text { inflammation, } \\
\text { acute ischemia, } \\
\text { necrosis }\end{array}$ \\
ECV & diffuse fibrosis & $\begin{array}{l}\text { amyloid, necrosis, } \\
\text { scar }\end{array}$ \\
iron, hemorrhage & $\begin{array}{l}\text { subacute } \\
\text { inflammation }\end{array}$ & $\begin{array}{l}\text { acute } \\
\text { inflammation, } \\
\text { acute ischemia, } \\
\text { necrosis }\end{array}$ \\
& $\begin{array}{l}\text { T2* } \\
\text { iron, hemorrhage, } \\
\text { stress-induced } \\
\text { ischemia }\end{array}$ & & \\
\hline
\end{tabular}

10)There is currently no specific recommended/ preferred analysis software package. The image reader should be trained with the local standards and with the analysis software package of choice and be aware of and familiar with the appearance of artifacts.

11)Sensitivity of mapping techniques to confounders such as heart rate and magnetic field inhomogeneities should be considered during interpretation.

\section{Reporting}

1) For clinical reports, the type of pulse sequence, reference range, and type/dose of gadolinium contrast agent (if applied) should be quoted.

2) Mapping results should include the numerical absolute value, the Z-score (number of standard deviations by which the result differs from the local normal mean; if available), and the normal reference range.

3) An interpretation should be given as normal, mild, moderate, or severe increase/decrease.

4) Best practice is defining the severity of deviation based on prognostic data. If such data are not available (as is the case for most applications of cardiac mapping) and no published suitable scheme 
is available, the findings may be classified as mild, moderate, and severe, referring to tertiles of the known spectrum of disease severity.

\section{Rationale and diagnostic potential of parametric mapping techniques}

Cardiac mapping techniques extend the diagnostic capabilities of CMR by enabling the quantification of CMR signal changes on an absolute scale. Conventional CMR techniques require a reference tissue (intracardiac: remote myocardium, or extracardiac: skeletal muscle) to detect alterations of myocardial tissue composition. The direct quantification of myocardial tissue properties effectively eliminates the need for such a reference tissue, which makes parametric mapping the first CMR tool that allows for direct assessment of diffuse myocardial disease.

The different cardiac mapping techniques provide specific parameters of the myocardium (native T1, T2, $\mathrm{T} 2 \%$, and ECV). While changes of these parameters are not specific for single diseases, they might serve as valuable biomarkers in the context of specific clinical scenarios, as changes of these parameters can be grouped into different patterns depending on the underlying pathology and reflect significant alterations in myocardial tissue composition (Fig. 3), Table 5.

As with any novel diagnostic approach, much of the research initially published in the field of parametric mapping was centered on advances in acquisition methodology. With a multitude of fast and robust mapping techniques described in the literature, some of which are commercially available on modern CMR systems. There is now growing evidence on the clinical value of CMR myocardial mapping from large-scale clinical outcomes trials. In principle, CMR myocardial mapping techniques hold important potential for making diagnosis, risk-stratifying, and monitoring therapy. Thus, parametric mapping can be regarded as a natural extension of comprehensive CMR protocols for the assessment of myocardial disease.

\section{Available evidence}

CMR mapping of T1, T2, T2*, and estimation of ECV in the clinical setting has intensively been studied over the past decade. Until very recently, the necessary pulse sequences and post-processing software have not been commercially available. Scientific developments in the field have mainly been driven by single large CMR centers, while evidence from multicenter trials is limited. Thus, current levels of evidence in the field mainly rely on single-center studies and opinions of experts who perform CMR mapping sequences in clinical routine for several years.
The present recommendations intend to introduce the concept of development steps towards the clinical application of T1, ECV, T2, and T2* mapping. These steps are defined by the available evidence supporting the clinical use of particular mapping sequences for the assessment of distinct disease patterns.

The proposed levels of utility are A) established applicability and usefulness which has been demonstrated by several clinical trials performed by more than one CMR center; B) emerging utility, defined as applications that have been proven useful in only one center; and C) potential applications, i.e. applications that have so far only been tested in experimental settings or animal models.

\section{Physical and technical background}

\section{Principles of measuring magnetic relaxation times}

The most common method for measuring relaxation times is to acquire a series of images in which the time to readout after inversion had been varied over a sufficiently wide range. The relaxation time can then be calculated on a pixel-by-pixel basis by fitting the image intensity of the series against the parameter that was used to vary the relaxation time weight. This pixel-wise relaxation time fitting needs to meet two conditions in order to be accurate and to avoid bias: 1) the variation in weight of other factors (different relaxation times, diffusion, etc.) is negligible or corrected for, and 2) there is negligible physical displacement between the images in the series. In the case of $\mathrm{CMR}$, cardiac and respiratory motion make meeting both conditions more challenging: avoiding motion artifacts limits the pulse sequence choices. Cardiac motion is normally avoided by only acquiring for a sufficiently short duration at mid-systole or enddiastole, while respiratory motion can be avoided or compensated for through breath holding or navigator gating, respectively. Any residual image-to-image displacement (shifts due to respiration or myocardial size differences due to contraction of the heart) can potentially be corrected through affine or non-rigid image registration after the acquisition and before pixel-by-pixel fitting. After the maps have been generated, several segmentation options are available: 1) the entire LV myocardium is segmented to establish an average value or perform a threshold-based analysis to determine areas of abnormality as a percentage of the LV myocardium, 2) the septal region is segmented, 3) the myocardium is divided into 16 segments as defined by the American Heart Association guidelines, or 4) ROIs are drawn in regions of pathology and healthy (remote) tissue for comparison. Option 2 (septal assessment) is mandatory for T2* mapping due to frequent artifacts in other regions of the myocardium (especially off-resonance close to the 
air-tissue interface), and might also be preferable for the assessment of diffuse myocardial disease by $\mathrm{T} 1$ and $\mathrm{T} 2$ mapping because of a high level of robustness and ease of omitting contamination from epicardial fat.

\section{Acquisition and processing strategies $T 1$ mapping and ECV}

Several approaches for the quantification of myocardial $\mathrm{T}_{1}$ have been described. The majority of sequences utilize multiple single-shot bSSFP acquisitions, using inversion preparation (MOLLI [3-5], ShMOLLI [6], ANGIE [7], STONE [8]), saturation preparation (AIR [9], SASHA [10], SAP-T1 [11]) or a combination of the two (SAPPHIRE [12]).

The modified Look-Locker [13] inversion recovery (MOLLI) sequence is widely used for myocardial $T_{1}$ mapping $[3,5,14]$. MOLLI offers excellent T1 precision, although its T1 assessment is sensitive to magnetization transfer (MT) effects [15], $\mathrm{T}_{2}$, flip angle, inversion pulse efficiency, heart rate and off-resonance, which reduces its accuracy [15-18]. Variants of MOLLI, defined by the number of images (or seconds) in a set (i.e. acquired following a given preparation pulse) and the number of recovery heartbeats (or seconds) between sets, have been optimized based on targeted ranges of $\mathrm{T} 1$ values and heart rates [16]. The MOLLI 5(3)3 [16] and the Shortened MOLLI (ShMOLLI) variants have emerged as approaches that require reduced scan time at a modest noise penalty [6], with little impact on their practical performance in direct comparison to the original MOLLI scheme [16, 19-22].

Saturation-based approaches typically use a single image acquisition per preparation pulse, minimizing the complex magnetization history of the Look-Locker approaches, and largely eliminating sensitivity to MT, T2, heart rate and off-resonance effects at the price of reduced precision $[10,15,16]$. SAturation Pulse Prepared Heart-rate-independent Inversion REcovery (SAPPHIRE) uses a hybrid of saturation and inversion pulses to improve precision of the saturation-recovery approach while maintaining accuracy [12].

Researchers continue to address the challenges of optimizing accuracy and precision with new acquisition and processing methods, for example, with the use of Bloch equation-based T1 corrections for MOLLI acquisitions [23-25] and a free-breathing version of SASHA with improved precision and contrast [26]. Whole heart T1 mapping with 3D ANGIE [7] or Slice-interleaved T1 mapping (STONE) [8] have illustrated full T1 map coverage of the LV with free-breathing acquisitions. In patients with atrial fibrillation, systolic data acquisition might be more robust than diastolic readout, but yields lower $\mathrm{T} 1$ values $[27,28]$.
T1 mapping can be used to estimate the myocardial ECV, a validated surrogate marker of fibrosis [29-31] in the absence of confounders (e.g. infiltration), based on the change in $\mathrm{T} 1$ values following the injection of conventional extracellular T1-shortening agents (e.g. GdDTPA) [22]. ECV estimation requires repeated T1 mapping acquisitions at baseline (native T1) and postcontrast delivery (typically $>10$ min post-contrast to approach steady-state conditions). Briefly, the change in 1/ T1 (R1) in the tissue and blood pool is used to determine contrast agent concentrations, the ratio of which yields an estimation of ECV, following a correction for red blood cell density in the blood pool (hematocrit). Recently, a simplified approach for the assessment of ECV was presented where the hematocrit was estimated from native values of blood pool T1 ("synthetic ECV") [32]. Preliminary data suggest that synthetic ECV values correlate with hematocrit-derived $\mathrm{ECV}$, which might allow for abstaining from laboratory tests of hematocrit for the assessment of ECV in the future [33].

For T1 mapping acquisition methods, T1 values are estimated by fitting a T1 recovery curve to each pixel in a series of images with different degrees of T1 recovery using a two- or three-parameter fit [3]. Therefore, motion between images will adversely impact T1 measurements and should be corrected $[34,35]$. Methods for the quantification of variability (e.g. quality control maps) in $\mathrm{T} 1$ and ECV values in the calculated maps have been described [36].

\section{T2 mapping}

In the presence of edema, myocardial $\mathrm{T} 2$ will increase. T2 weighted CMR imaging is commonly used to assess myocardial inflammation. However, image quality, reproducibility and subjective assessment of T2 weighted images have been limiting factors in its clinical adoption. To overcome these challenges, regional myocardial T2 mapping has emerged to directly quantify local myocardial inflammation and edema. Few studies have investigated the value of T2 mapping in other myocardial diseases [37]. T2 mapping is generally performed by pixel-wise fitting for a T2 decay curve of a series of T2weighted images. These source images can be acquired by a TSE sequence with varying echo time [38], a bSSFP or spoiled gradient echo (GRE) sequence with an initial $\mathrm{T} 2$ preparation module [39-42], or a sequence scheme that combines spin echo excitation with gradient echo readout (GraSE) [43]. In these T2 mapping sequences, images are acquired with different echo times (e.g. 0, 25, and $50 \mathrm{~ms}$ ) [39] and are used to estimate the T2 values using a two-parameter or three-parameter fit model [44]. Despite the potential of T2 mapping to replace T2weighted imaging for assessment of inflammation and edema due to its quantitative nature and higher 
robustness, confounders such as sensitivity to T1 and off-resonance effects have to be considered. Recent studies have thus focused on quantifying the reproducibility [45-50], robustness against artifacts [51], and the influence of motion correction $[45,52]$. At the same time, several challenges of myocardial T2 mapping have been overcome by proposing faster image acquisition, reducing bias [44], and increasing spatial coverage [53-55].

\section{T2* mapping}

T2* relaxation mapping is usually based on GRE sequences and forms the basis for numerous CMR applications [56]. In particular, T2* quantification is currently the method of choice for myocardial tissue iron assessment [57]. In this scenario, images are typically acquired at 8 different echo times ranging from 2 to $18 \mathrm{~ms}$. For cardiac imaging, a segmented GRE sequence combined with ECG gating allows all echo images to be acquired in a single breath-hold. Currently, both a bright blood [58] and a black blood [59] technique are validated and widely used clinically $[60,61]$. For the bright-blood technique, images are acquired immediately after the R-wave to reduce artifact because of blood flow and myocardial wall motion. For the black-blood technique, a double inversion recovery pulse is used to null the signal from blood, and data acquisition is extended to late diastole with minimal cardiac motion. In comparison, the black-blood technique produces less bias and reduced interobserver variability and is hence recommended if available [62].

For the measurement of myocardial $\mathrm{T} 2{ }^{*}$ in vivo, a midventricular short axis slice is acquired and a homogeneous ROI is defined encompassing both sub-epicardial and subendocardial regions, as iron is preferentially stored in the sub-epicardial compared with the sub- endocardial layers [62]. The analysis is restricted to the septum to reduce susceptibility artifacts. A multi-slice $\mathrm{T} 2 *$ approach has also been proposed, which may have advantages for the evaluation of myocardial hemorrhage in acute myocardial infarction by providing whole-heart coverage. The singleslice technique based on the septal analysis remains accepted practice [63].

\section{Quality control}

Changes in CMR system hardware and software may lead to changes in mapping sequence parameters (see Normal values). During image acquisition, mis-triggered heart beats, motion (cardiac and respiratory), and off-resonance or other artifacts may lead to inaccurate measured relaxation times (see [64], electronic supplementary material). When possible, breath-held acquisitions with data collection during the quiescent period the cardiac cycle, typically middiastole, is preferred. Pixel-by-pixel parametric maps still benefit from breath-holds, even if motion-correction algorithms are used, as motion correction algorithms can only correct in-plane but not through-plane motion. These algorithms can incorrectly co-register anatomy, and corrupt image quality and accuracy [48], so it is imperative to view the motion-corrected raw images to ensure that the motion correction had worked properly. The operator should ensure that there are no mis-triggered or skipped heart beats during data acquisition. Once acquired, the user should immediately inspect the raw images, quality control maps (e.g. $\mathrm{R}^{2}$ or error), if available, and the parametric maps to assess for quality, reacquiring images as necessary. For instance, motion may manifest as obvious diaphragmatic motion or variation in cardiac phases on the raw images, areas of poor curve fit on quality control maps, or blurring of tissue borders on T1/T2 maps. For T2* measurements, poor breath holding and cardiac motion typically manifests as ghosting, and may significantly affect image and data quality. Offresonance artifacts may be particularly prominent at $3 \mathrm{~T}$, and the use of a local box shim is essential to reduce offresonance variation across the heart.

\section{Normal values}

In healthy subjects, myocardial tissue exhibits a very uniform composition and thus possesses very regular magnetic properties. As a result, native T1, T2, and T2* values from normal myocardium are highly reproducible and show relatively narrow ranges when acquired under the same conditions.

In practice, the term "same conditions" becomes highly relevant. If these conditions change, it becomes impossible to judge if changes in tissue parameters results are due to a pathologic state or simply to a change in the way the measurement was performed.

The conditions for parametric mapping can be divided into two areas:

\section{Physical/biological confounders. These include} magnetic field strength and temperature, meaning that native $\mathrm{T} 1, \mathrm{~T} 2$, and $\mathrm{T} 2 \%$ values are not directly comparable between $1.5 \mathrm{~T}$ and $3 \mathrm{~T}$, T1 will increase by approximately $1 \%$ for every $1{ }^{\circ} \mathrm{C}$ increase in body temperature [65], and T2 shortens with increasing temperature [66]. Temperature dependency is usually neglected as extremes of body temperature (pyrexia, hypothermia) are not typical features of patients undergoing a CMR scan, but nonetheless should be considered in the unusual event that a febrile (or hypothermic) patient undergoes CMR parametric mapping.

2. Methodological confounders. The acquisition and processing of parametric maps require several technical steps. There are multiple technical options for each of these steps, each of those introducing different types and degrees of error to the 
measurement. For example, the use of inversion recovery Look-Locker based acquisition schemes for T1 mapping introduces a significant negative offset to $\mathrm{T} 1$ values as compared to saturation recovery sequences; the use of insufficient waiting time for signal recovery in inversion recovery $\mathrm{T} 1$ methods and T2-prepared mapping can introduce heart rate dependence; and low flip angles can lead to low SNR in all approaches.

This problem is complicated by the fact that there is no reference technique to determine the "real" myocardial relaxation times in-vivo, as in-vitro reference techniques cannot be applied to live human hearts and hearts from other species (that might be subjected to several hours of scanning) do not exhibit the exact same normativevalues as human hearts.

As a consequence, native parameter values should only be compared to other parameter values if they are obtained under similar conditions. In other words, the acquisition scheme, field strength and processing approach should be the same, and the results should be reported along with corresponding reference ranges for the given methodology (see Reporting). This situation actually resembles that of other biomarkers including serologic tests, where reference values of the local laboratory are usually provided along with the results sheet. In the future, it might become possible to normalize native parameter results to a "standard parameter" based on phantom or software calibration methods [67].

Apart from these variations caused by confounders, there are subtle differences in myocardial T1 and T2 (but not $\mathrm{T}^{*}$ [68]) that are related to gender and (less so) age. In 1231 participants of the Multi-Ethnic Study of Atherosclerosis (MESA) aged 54 to 93 years, women showed stable native $\mathrm{T} 1$ throughout different age groups whereas men had lower native $\mathrm{T} 1$ at 54 to 63 years that increased in higher age groups and approached that of women in the group of $>84$ years [69]. In a study of 342 healthy subjects, native $\mathrm{T} 1$ values of women were higher up to the age of 45 years [70]. Similarly, a study in 74 healthy subjects revealed higher myocardial T2 in women and in subjects with greater age $[47,71]$. Further insights are expected from on-going other populationbased studies [72]. Taken together, the magnitude of gender- and age-related differences for myocardial relaxation times in these studies reached approximately 0.5 standard deviations from the normal mean. While these effects seem negligible in diseases with alterations of high magnitude (e.g. amyloidosis), they become relevant if pathologic processes with more subtle impact on native relaxation times such as diffuse myocardial fibrosis are of interest. As a consequence, non-specific normal ranges might be appropriate for high-magnitude disease states, while the assessment of low-magnitude pathologies requires the use of granular normal ranges according to gender and to a lesser extent age, which are currently not available for all acquisition approaches.

\section{Clinical applications: Current state Mechanisms of myocardial injury}

$\mathrm{T} 1, \mathrm{~T} 2$, and $\mathrm{T} 2 *$ times of a given pixel in a respective parametric map represent a composite signal of the corresponding tissue voxel. Changes of myocardial tissue composition lead to alterations of T1, T2, and T2* times. Such changes usually occur in the same direction (i.e. shorten or lengthen) for all three magnetic properties but might not reach the same degree in all of them, depending on the underlying process. ECV behaves different in that it only reflects alterations of the extracellular component of the myocardium. Table 10 gives an overview on principal directions and degrees of changes in parameters depending on pathology.

\section{Acute myocardial disease Acute ischemic injury}

As acute myocardial edema develops in areas of acute ischemia and infarction, native $\mathrm{T} 1$ and $\mathrm{T} 2$ relaxation times prolong, whereas post-contrast $\mathrm{T} 1$ time shortens, compared to remote myocardium [14, 73, 74]. Both native $\mathrm{T} 1$ and $\mathrm{T} 2$ mapping correlate well with the area-at-risk measured by microspheres in animal studies [75], and may be used to delineate the area-at-risk and determine myocardial salvage in clinical applications [76, 77]. With the development of significant interstitial edema and other changes in the vasculature, ECV is expanded in the areas of acute myocardial injury and infarction [78]. Compared to T2-weighted imaging, native T1 mapping was superior in detecting areas of injury in non-ST elevation myocardial infarction (NSTEMI), and similar in STEMI [79]. Furthermore, native $\mathrm{T} 1$ and $\mathrm{T} 2$ mapping may provide prognostic information through the identification of the infarct core [80] and intra-myocardial hemorrhage [81], both of which are associated with an adverse prognosis. Post-contrast T1 and ECV changes have been identified in the remote myocardium early in the course of acute infarction [82-84], suggesting that adverse cardiac remodeling may commence at the time of infarction and is not simply a consequence of longer term hemodynamic stress. T2 mapping may be used to track the resolution of myocardial edema, and $\mathrm{T} 2 *$ imaging may be used to assess for intramyocardial hemorrhage and reperfusion injury post infarction [85]. 


\section{Acute inflammation}

Increases in myocardial free water content, as occurs in acute myocardial edema and inflammation, prolong $\mathrm{T} 1$ and T2 relaxation times [73, 86]; where myocardial edema is extra-cellular, this will also expand the interstitial space and, hence, ECV. Both T1 and T2 mapping are sensitive to detecting acute myocardial edema and inflammation in animal models $[73,75,86]$ and in clinical patients who present with various forms of acute myocardial injury, including myocardial infarction, stress-induced (a.k.a. Tako-tsubo) cardiomyopathy and myocarditis [64, 79, 87-89]. Mapping techniques possess a number of technical advantages over conventional T2-weighted imaging for detecting myocardial edema and inflammation, and have demonstrated superior diagnostic performance in the clinical setting [64, 79, 87, 89]. T1, ECV and T2 mapping have clinical utility in the diagnosis of acute myocarditis, shown by a number of clinical studies, and may be used in conjunction with the Lake Louise Criteria [90, 91]. Additionally, mapping techniques are sensitive to less acute presentations of inflammation, and are able to detect subclinical forms of myocarditis as part of systematic inflammatory diseases, such as rheumatoid arthritis, lupus erythematosus, systemic sclerosis, pheochromocytoma, human immunodeficiency virus infection and cardiac sarcoidosis [92-97]. Mapping techniques may also have emerging roles in the diagnosis of cardiac transplant rejection [98], and differentiation of athlete's heart from dilated cardiomyopathy, which may have an inflammatory component detectable using parametric maps [99].

\section{Heart failure with reduced or preserved ejection fraction Aortic stenosis}

While the key measure determining the need for valve replacement in aortic stenosis is the degree of valvular obstruction, the myocardium also undergoes progressive changes that can lead to deteriorating cardiac performance and increased morbidity and mortality [100]. The degree of LV hypertrophy in aortic stenosis is independently associated with a higher rate of cardiovascular events [101], and recent T1 mapping data suggest that myocardial ECV is a stronger predictor of adverse cardiovascular outcomes than the extent of LV hypertrophy. A number of T1 mapping techniques have already been shown to correlate with the degree of histological interstitial fibrosis in aortic stenosis patients [102, 103]. Compared to healthy controls, patients with aortic stenosis have a higher ECV [30], suggesting a maladaptive response to pressure overload. In these patients severe diastolic dysfunction is associated with higher ECV levels, implying a mechanistic link between interstitial fibrosis and myocardial stiffness. The notion that pathological LV remodeling in aortic stenosis is at least in part driven by increasing levels of interstitial fibrosis suggests that in the future $\mathrm{T} 1$ mapping may play a role in predicting future cardiovascular outcomes in aortic stenosis, or even help to time intervention. Although the principle determinant of regression of hypertrophy following aortic valve replacement for severe aortic stenosis may be a reduction in cell volume rather than regression of interstitial fibrosis [30], studies are currently underway aimed at assessing the role of ECV measurement in the selection of patients for aortic valve replacement. Furthermore, it has been suggested that coronary vasodilation may also contribute to increased native $\mathrm{T} 1$ via intravascular volume expansion in severe aortic stenosis [104].

\section{Arterial hypertension}

Similar to the response to pressure overload in aortic stenosis, CMR T1 mapping studies in patients with arterial hypertension have demonstrated small degrees of expansion in ECV that parallels the development of LV hypertrophy [105]. Furthermore, the development of ECV expansion as a consequence of hypertension is associated with reduced cardiac performance, suggesting a mechanism by which hypertrophy in hypertension may evolve into a more malignant phenotype $[106,107]$. In addition, T1 mapping may play a diagnostic role in discriminating patients with LV hypertrophy due to hypertrophic cardiomyopathy from those with hypertrophy secondary to arterial hypertension [108]. This distinction is not merely academic, as hypertension is a common finding in the community and there are important differences in the management of patients with hypertrophic cardiomyopathy compared to those with hypertensive heart disease. Finally, of great interest would be the demonstration of regression of interstitial fibrosis following antihypertensive therapy. In one study, following marked blood pressure reduction in a cohort of hypertensive patients who underwent renal denervation, there was a significant reduction in the blood-tissue partition coefficient, lambda (a surrogate of ECV), in those who underwent renal denervation versus controls [109].

\section{Amyloid disease}

Two types of amyloidosis commonly infiltrate myocardium: immunoglobulin light-chain derived (AL) and transthyretin (ATTR) amyloid. While these two types have different natural history and prognosis, cardiac involvement drives outcome and therapeutic choices in both. Early recognition and therapy are critical in AL amyloidosis when cardiac involvement is detected. CMR with LGE has been shown to be a valuable tool in cases with typical subendocardial tramline pattern, which is replaced by transmural RV and LV enhancement at later stages. 
However, atypical patterns have been described and renal impairment restricting contrast use is common in amyloid. Native T1 mapping may allow for making the diagnosis of cardiac amyloidosis without the need for gadolinium contrast application, although the normal $\mathrm{T} 1$ in renal disease is not yet well defined [110-112]. ECV appears to be a surrogate marker for the amyloid burden and carries prognostic value [113]. In early disease, native $\mathrm{T} 1$ and ECV are elevated before LGE appears, although these changes are initially non-specific and thus only clinically useful when the pre-test probability is high. Once subendocardial LGE appears, ECV elevation in remote areas begins to be diagnostic, as diffuse fibrosis rarely increases the ECV above $40 \%$. ECVs of $>55 \%$ appears in transmural LGE. The T1 and ECV are subtly divergent in established AL vs. ATTR with a higher ECV in ATTR, a higher native T1 in AL, and greater cell volume expansion in ATTR [114]. Particularly in the elderly, occult ATTR amyloid confounds other diseases such as heart failure, hypertrophic cardiomyopathy, and aortic stenosis, although bone tracer scanning appears more sensitive [115]. ECV can be used to track therapy in amyloid [116], and might be able to track amyloid regression.

\section{Anderson-Fabry disease}

Anderson-Fabry disease is a rare $\mathrm{X}$-linked lysosomal storage disease that causes LV hypertrophy and eventually fibrosis and heart failure. The underlying pathology is intra-cellular accumulation of glycosphingolipid. Native T1 in this disease is low, unlike any other cause of hypertrophy except for iron overload (see Iron overload), in around $85 \%$ of all subjects with LV hypertrophy, though to be directly related to sphingolipid storage $[117,118]$. This is highly characteristic and diagnostic, despite the rarity of Anderson-Fabry and consequent low pre-test probability. T1 is low in around half of patients with Anderson-Fabry even in the absence of hypertrophy, making T1 mapping a useful test for early cardiac involvement and raising the possibility of early therapy to prevent the downstream events and overt disease.

Patients with Anderson-Fabry frequently show a characteristic mid-wall LGE pattern in the basal inferolateral wall, which often appears thin. Recent data suggest that in this area, in contrast to LGE from other origin, there is an increased native $\mathrm{T} 1$ and $\mathrm{T} 2$ even in the absence of wall thinning, with the T2 elevation corresponding to elevated levels of blood troponin. This suggests that the lesions on LGE may reflect chronic, active inflammation [119]. This important finding may be a critical link of underlying mutation to a final fibrotic phenotype and may point to a pathway common to other non-ischemic LGE findings and disease development.

\section{Iron overload}

Cardiac iron overload is a serious condition, caused either by repeated blood transfusions for anemia (e.g. in thalassemia major) or increased intestinal iron absorption (e.g. hereditary hemochromatosis). Iron overload leads to severe heart failure and lethal arrhythmias but can be treated effectively if diagnosed early. T2* mapping is an accurate and reliable method for the quantification of cardiac iron load [120]. It is reproducible across different CMR systems [121, 122], and identifies patients at risk for heart failure or arrhythmia from myocardial siderosis [123]. Furthermore, T2* mapping can be used to monitor disease progression and therapy [124$126]$, and currently is the only parametric mapping technique that is recommended in disease-specific clinical guidelines [62].

Recently, T1 mapping has also been evaluated for the assessment of cardiac siderosis, and compared with T2 and $\mathrm{T}_{2}^{*}$ in a substantial population with and without cardiac iron overload [127], showing considerable scatter between techniques. In patients with only mild increases of cardiac iron, non-contrast T1 mapping showed superior reproducibility as compared to $\mathrm{T} 2 *$ measurements [128], but there might be little relevance of this finding in significant iron overload states causing heart failure.

ECV may be increased in patients with thalassemia major and is associated with cardiac iron overload [129]. However, ECV correlated significantly with lowest historical $\mathrm{T}^{*}$ measurements but not with systolic function. Thus it is currently unknown whether ECV has a role in the management of cardiac siderosis patients.

\section{Diffuse myocardial fibrosis and cardiac remodeling}

Myocardial fibrosis occurs in a continuum from mild to severe where excess collagen (concentration) appears in the myocardial interstitium [130]. Accordingly myocardial native $\mathrm{T} 1$ and ECV increase, whereas post contrast T1 decreases [2]. ECV is well suited to measure interstitial expansion occurring with fibrosis (or amyloidosis) with extracellular gadolinium-based contrast agents. ECV simply quantifies the interstitial presence of gadolinium relative to the plasma. Accordingly, histologic validation data overall show best agreement with ECV compared to other T1 metrics based on R2 values (i.e., the proportion of variation in a variable explained by another variable) $[131,132]$. Despite the potential confounding introduced by capillary rarefaction or myocardial edema (since myocardial gadolinium presence includes the myocardial vasculature), most validation studies report high $R 2$ values $\geq 0.6[29,103,133-138]$. The intra-myocardial vascular compartment is another potential confounder in the setting of vasodilation (e.g., adenosine $[104,139,140])$, so the clinical context for ECV measures must be known for optimal interpretation. Since LGE detection of fibrosis 
depends on its spatial heterogeneity, LGE is not designed for quantifying fibrosis in noninfarcted myocardium and is not validated as a quantitative metric for this purpose. Nonetheless, LGE can often identify cardiac amyloidosis (see above) [113]; thus LGE can assist discrimination of the cause of elevated ECV, i.e., myocardial fibrosis versus cardiac amyloidosis.

ECV dichotomizes the myocardium into its primarily cellular compartment and predominantly interstitial compartment (including the myocardial vasculature) [2]. While myocardial fibrosis may follow myocyte loss due to various injuries, it also may occur with primary fibroblast activation. The positive correlation between myocardial fibrosis (whether by ECV or histology) and LV mass suggests significant primary fibroblast activation since myocyte loss would decrease LV mass [132]. This information is relevant when appraising potential therapeutic targets.

Emerging data reveal that many cardiac insults culminate in myocardial fibrosis, and the extent of fibrosis can vary across disease categories [78]. The extent of myocardial fibrosis regardless of cause or disease category then appears to govern vulnerability to adverse outcomes (death or heart failure) [141]. ECV appears to reflect the extent of myocardial fibrosis and has been validated against collagen volume fraction $[29,103,133-138]$. ECV has been shown to be reproducible [136, 142-147], predict outcomes [148-155], and provide "added prognostic value" manifest by reclassification metrics [141]. Thus, ECV quantification of interstitial expansion remains a powerful tool to investigate myocardial remodeling, especially when combined with ancillary clinical data.

\section{Primary cardiomyopathy}

Dilated cardiomyopathy and hypertrophic cardiomyopathy are associated with the development of diffuse myocardial fibrosis. In both groups of patients, native T1 was found to be increased not only in areas corresponding to LGE but also in areas without LGE, hence suggesting that native T1 can detect areas of tissue pathology beyond those detected by LGE [156]. In patients with dilated cardiomyopathy, high native myocardial $\mathrm{T} 1$ is associated with an increased risk for cardiovascular events and heart failure [157]. Furthermore it could be shown for both dilated cardiomyopathy [158] and hypertrophic cardiomyopathy [159] that ECV is increased not only in patients with typical phenotype, but also in asymptomatic relatives without clinical findings but with positive phenotype. Whether ECV provides additional information in these patients for the prediction of ventricular arrhythmia is under investigation. There are no data on the diagnostic value of parametric mapping in patients with arrhythmogenic right ventricular cardiomyopathy (ARVC). The fibrofatty replacement, which is a typical finding in this disease, should theoretically lead to alterations of both native T1 and ECV. However, the thin RV free wall prevents the application of breathhold parametric mapping techniques, which do not provide high-enough spatial resolution. Respiratory-gated segmented techniques might circumvent this problem in the future [7].

\section{Valvular disease}

Fibrotic remodeling as a consequence of chronically increased afterload has long been recognized. Even young patients with aortic stenosis exhibit increased ECV, which correlates with the degree of diastolic dysfunction [160]. In addition to the cardiac remodeling driven by pressure overload, volume overload driven by valvular regurgitation is also associated with adverse cardiac remodeling. In contrast to stenotic valvular lesions, most patients with valvular regurgitation develop significant cardiac remodeling prior to the development of symptoms; therefore the role of CMR in characterizing subclinical myocardial changes is of particular importance. In patients with chronic aortic regurgitation, shorter post-contrast $\mathrm{T} 1$ time, consistent with diffuse myocardial fibrosis, is present in myocardial segments with impaired function [161], again suggesting a relationship between cardiac dysfunction and interstitial fibrosis. More recently, similar findings have been demonstrated with ECV in patients with mitral regurgitation [162], where changes in ECV were linked with early cardiac remodeling and reduced cardiovascular performance. Taken together these studies suggest a potential future role for CMR T1 mapping in the identification of subclinical myocardial changes due to volume overload, which may help guide future interventional strategies for regurgitant valve lesions.

\section{Ischemic heart disease}

Besides the severe alterations of native $\mathrm{T} 1$ seen in the acute stage (Acute ischemic injury), myocardial infarction also causes changes of native T1 in the chronic stages of the disease. However, the heterogeneous nature of these changes both within and in between subjects limit the sensitivity of native $\mathrm{T} 1$ for the detection and - even more so - the quantification of the extent of chronic myocardial infarction in a given individual $[14,163]$. An increase of native $\mathrm{T} 1$ has also been reported for remote areas of hearts with acute myocardial infarcts (see Acute ischemic injury), and was associated with LV remodeling and adverse cardiac events [164]. Recently, characteristic patterns of pathologic $\mathrm{T} 1$ response to adenosine stress have been demonstrated in patients with significant coronary artery stenosis using native T1 mapping $[139,140]$. Apart from direct effects of impaired myocardial perfusion on $\mathrm{T} 1$, additional mechanisms that might contribute to these 
patterns include blood oxygenation level dependent (BOLD) and arterial spin labelling (ASL) effects [165]. Further studies are necessary to answer the question if native T1 mapping can be used as a non-contrast stress perfusion test for the assessment of myocardial ischemia.

\section{Congenital heart disease}

In congenital heart diseases, volume and/or pressure overload are important factors in alterations of myocardial structure and function [166]. In adolescents and adults with Tetralogy of Fallot, for example, the presence of volume overload due to pulmonary regurgitation is associated with expansion of ECV, which in turn is associated with a higher incidence of cardiac arrhythmia [167]. Although Tetralogy of Fallot is a "right heart disease", LV ECV is elevated as well [168, 169]. Pediatric Tetralogy of Fallot patients from a contemporary era enjoy an overall better myocardial health than previous surgical generations, but an association of both ECV and native $\mathrm{T} 1$ in the $\mathrm{LV}$ with exposure to cardiopulmonary bypass more than 10 years earlier remains a concern and highlights the need for improved cardioprotection [168]. Furthermore, chronic hypoxemia and genetic disposition have been associated with adverse myocardial remodeling in repaired and unrepaired malformations of the heart. Nearly all CMR T1 mapping studies in Tetralogy of Fallot have demonstrated higher ECV and/or native $\mathrm{T} 1$ values in females [167]. This finding together with worse RV function and exercise tolerance [170] suggests that we may need to monitor and treat females differently from males after Tetralogy of Fallot repair.

Patients with physiologically uni-ventricular hearts (so called "single ventricles") are at particular risk for developing ventricular dysfunction, especially when the dominant ventricle is of RV morphology, as in hypoplastic left heart syndrome [171]. Even at a young age, these patients have been demonstrated to develop elevated ECV, which is associated with reduced myocardial contractility. So far, the prognostic significance of this finding is not known.

Drug trials for the treatment of heart failure in congenital heart disease have been nearly unequivocally disappointing. Information on myocardial health from T1 mapping might potentially allow for a more personalized pharmacological approach. As pharmacologic heart failure therapies have been less effective in these situations than in acquired heart disease, interventional and surgical approaches play a major role. T1 and ECV mapping have been proposed as tools to guide decision-making and timing of such procedures in the course of the disease. For example, the goal of pulmonary valve replacement in post-repair patients with Tetralogy of Fallot may become preservation of myocardial health over and above restoring RV volume, which is currently at the center of timing for pulmonary valve replacement. Thus, longitudinal RV ECV assessments may prove helpful in making the decision when to restore pulmonary valve competency. However, RV disease is hard to assess with common breath-hold T1 mapping approaches as their spatial resolution approaches the low thickness of the RV wall. Even in patients with right ventricles that support the systemic circulation, the RV free wall has been deemed not measurable by T1 mapping [172]. This problem of insufficient spatial resolution is further aggravated in pediatric patients with small hearts. Therefore, while initial studies could demonstrate the diagnostic potential of $\mathrm{T} 1$ and ECV mapping in patients with RV disease, meaningful clinical applications seem to warrant the availability of high-resolution mapping techniques, which are based on non-breath hold (navigated) segmented acquisition strategies, examples of which are currently still restricted to research applications (ANGIE [7], SALLI [173]).

Information on the prognostic significance of parametric mapping in pediatric cardiomyopathies and myocarditis is scarce, and it remains unclear whether the experience in adults can be extrapolated to children. In patients with chronic Kawasaki disease, ECV is elevated, including in LGE-negative myocardial segments. It is highest in segments supplied by severely aneurysmal and/or obstructed coronary arteries and is associated with decreased myocardial blood flow and strain. Therefore, CMR T1 mapping may present an opportunity to improve risk stratification and monitoring in Kawasaki disease beyond coronary artery angiography and stress testing. In boys with Duchenne muscular dystrophy, heart failure treatment is typically started or intensified when myocardial dysfunction and/or LGE develop. However, even patients with normal LV ejection fraction and no LGE have elevated ECV and T1 values [174]. This observation suggests that T1 mapping, and perhaps native T1 more than ECV [175], may identify the onset of fibrotic remodeling earlier than LGE and ejection fraction, providing an opportunity for a more timely intervention.

\section{(Para-)cardiac masses}

Mapping techniques may be used for characterizing extramyocardial tissues, such as masses, pericardial effusion and fat, although current clinical evidence is sparse for these applications. For instance, cardiac masses with high fat content, such as lipomatous hypertrophy of the interatrial septum, may be readily apparent on T1 maps [176], demonstrating homogenous and characteristically low $\mathrm{T} 1$ values. Simple cysts and pericardial effusion, composed almost entirely of water, are expected to exhibit very long T1 and T2 relaxation times. Cystic lesions without communication with the systemic circulation and its vasculature will show unchanged $\mathrm{T} 1$ values post gadolinium administration. Masses that have a high water content and 
substantial vascularization (and therefore blood volume) also show long $\mathrm{T} 1$ and $\mathrm{T} 2$ relaxation times, and will have evidence of gadolinium contrast uptake (e.g. low T1 values post contrast) [177]. Due to bias caused by intramyocardial fat seen in inversion- and saturation-recovery T1mapping techniques, a wide range of native $\mathrm{T} 1$ values may be seen in tissues with fat content when a voxel is only partially occupied by fat $[178,179]$. It is also important to select mapping techniques that have been validated across a wide range of $\mathrm{T} 1$ and $\mathrm{T} 2$ values that include the ranges typically seen in the tissues types under study (e.g. cysts with very high $\mathrm{T} 1$ and $\mathrm{T} 2$ values), to avoid potential misdiagnosis due to underestimation of long relaxation times (e.g. cyst versus malignant tumor). More research and validation against tissue pathology are needed in this area before routine clinical applications for diagnoses.

\section{Knowledge gaps and areas for ongoing research Relaxometry assumptions}

The measurement of $\mathrm{T} 1, \mathrm{~T} 2$, and $\mathrm{T} 2 *$ relaxation time constants assumes a mono-exponential behavior on a macroscopic scale. This simplifying assumption has proven to be useful in clinical practice for differentiation of tissues and assessment of their state. However, the molecular composition of biological tissue is frequently more complex, leading to multiple compartments with chemical exchange (magnetization transfer) and finite diffusion distances. How the simplified model is affected by more complex molecular composition of tissue and pathology is unclear, and the clinical consequences of the simplified view needs to be studied for various scenarios.

\section{Confounding factors}

The measurement of a parameter of interest such as $\mathrm{T} 1$ may depend on other variables such as $\mathrm{T} 2$ or patient's heart rate (HR), and numerous other confounding factors. From a clinical perspective, the consequences of such bias are not fully understood. There are instances for which the confounding factor may make a disease more detectable. However, the interpretation of the shift from baseline normal is no longer clear. For example, an elevated T1 might be due to fibrosis, or might be due to confounding effect of elevated T2 arising from edema. In another example, a decrease in $\mathrm{T} 1 \mathrm{might}$ be due to increased iron concentration, or might be due to offresonance in the scanner center frequency. Some of the confounding variables are due to patient physiology, but others may be due to scanner adjustments or field inhomogeneities. The extent to which the desired measurement is confounded will depend on both the sequence design and specific protocol. Some of the confounding effects may be disentangled by multi-parametric measurements, or by calibrations scans, but these come at the expense of time and complexity.

\section{Partial volume effects}

Partial volume effects arise from a) the contamination of the desired signal by adjacent tissue such as blood pool or fat, or b) mixtures of tissue within the voxel of interest such as intramyocardial fat. Use of blood or fat suppression may help mitigate partial volume effects but may also affect the measurement of interest. The degree to which partial volume errors influence the measurement depends on aspects such as wall thickness or angulation of the slice prescription.

Myocardial tissue may be heterogeneous with variation on the scale of the voxel resolution. In this instance, the quantitative measurements are smoothed and represent an average, which may not accurately reflect the focal elevation or baseline normal values. For example, the focal elevation of T1 or T2 may depend on the slice thickness when the focal abnormality is thinner than the slice. This is compounded when ROIs are drawn which introduced further averaging. Thus the degree of heterogeneity and size of focal abnormalities is a factor influencing the quantitative measurement and depends on resolution and manner of measurement and reporting. For example, in assessment of iron overload, where the concentration of iron is heterogeneous, it is unknown whether there is clinical significance of the peak concentration and distribution in addition to the average value quantified in typical analysis. The influence of these effects on clinical assessment needs to be characterized.

\section{Post-processing}

The impact of the post-processing methodology (i.e. derivation of relaxation times from a set of source images) on the quality of parametric results can equal or sometimes even exceed that of the acquisition strategy. Consequently, advanced post-processing strategies have been investigated. For T1 mapping, several methods have been proposed that aim to optimize T1 estimation from MOLLI source images $[24,25]$. In $\mathrm{T}^{*}$ mapping, there is evidence that a commonly used offset model is fundamentally incorrect [180]. The truncation model is challenging in the presence of severe iron overload [181]. Researchers continue to address this challenge with novel methods. For example, an improved truncation model was extended to black-blood T2* mapping [182] and a noise-corrected mode was developed [183] to cope with both pixel-wise and region-of-interest (ROI)-based curve-fitting. Further studies are needed to identify the methods that provide the best diagnostic accuracy in clinical applications.

\section{Map analysis}

While the assessment of average parameter values of septal ROIs is regarded as appropriate for diffuse myocardial disease, conditions with patchy presentation such as myocarditis (which in some cases changes its distribution from focal 
to diffuse throughout the course of disease) might require more detailed analysis of regional behavior. Histogram analysis [6] and statistical analysis of tissue heterogeneity [184] have been proposed as means to detect and quantify different tissue populations within ROIs, and might be helpful in the analysis of inhomogeneous myocardial disease.

\section{Future directions}

Future developments in cardiac mapping will likely focus on standardization of data acquisition and post-processing, as well as on optimizing workflows. In parallel, the clinical utility of mapping will have to be carefully explored for various cardiovascular diseases to further define where mapping parameters can firmly establish diagnosis, guide therapeutic decisions, and predict prognosis. The robustness of mapping protocols and results will play an important role in its acceptance for clinical decision-making. Challenges include proprietary approaches of CMR system manufacturers, cost for software packages and the need for calibration of hardware and sequence settings.

On the technical side, accelerated image acquisition such as compressed-sensing techniques [185, 186] may allow for a significant shortening of scan times or for improving image quality or spatial resolution. Threedimensional (3D) mapping may allow for more complete coverage of the heart and a better characterization of complex regional distribution patterns of disease processes [53, 54, 187].

The combination of mapping with quantitative data from functional (cine) studies can be sufficient to differentiate diseases from physiologic adaptation of cardiac mass, shape and function such as in athlete's heart $[99,188]$.

As native $\mathrm{T} 1$ and $\mathrm{T} 2$ are sensitive to increased myocardial water content and myocardial blood volume, there is current interest to investigate the ability of vasodilatory stress $\mathrm{T} 1$ and $\mathrm{T} 2$ mapping to detect ischemia without the need for exogenous contrast agents as a novel application of mapping techniques [139, 140].

Several techniques have also appeared that map multiple relaxation times simultaneously (MR fingerprinting) [189-191], with the double advantage of saving time and removing confounders introduced by the interaction of relaxation times. Although the precision, bias, and reproducibility of such techniques need to be carefully quantified, these new techniques augment the available arsenal for various challenging imaging conditions, such as those found in patients with arrhythmia, patchy disease patterns or the inability to perform breath holds.

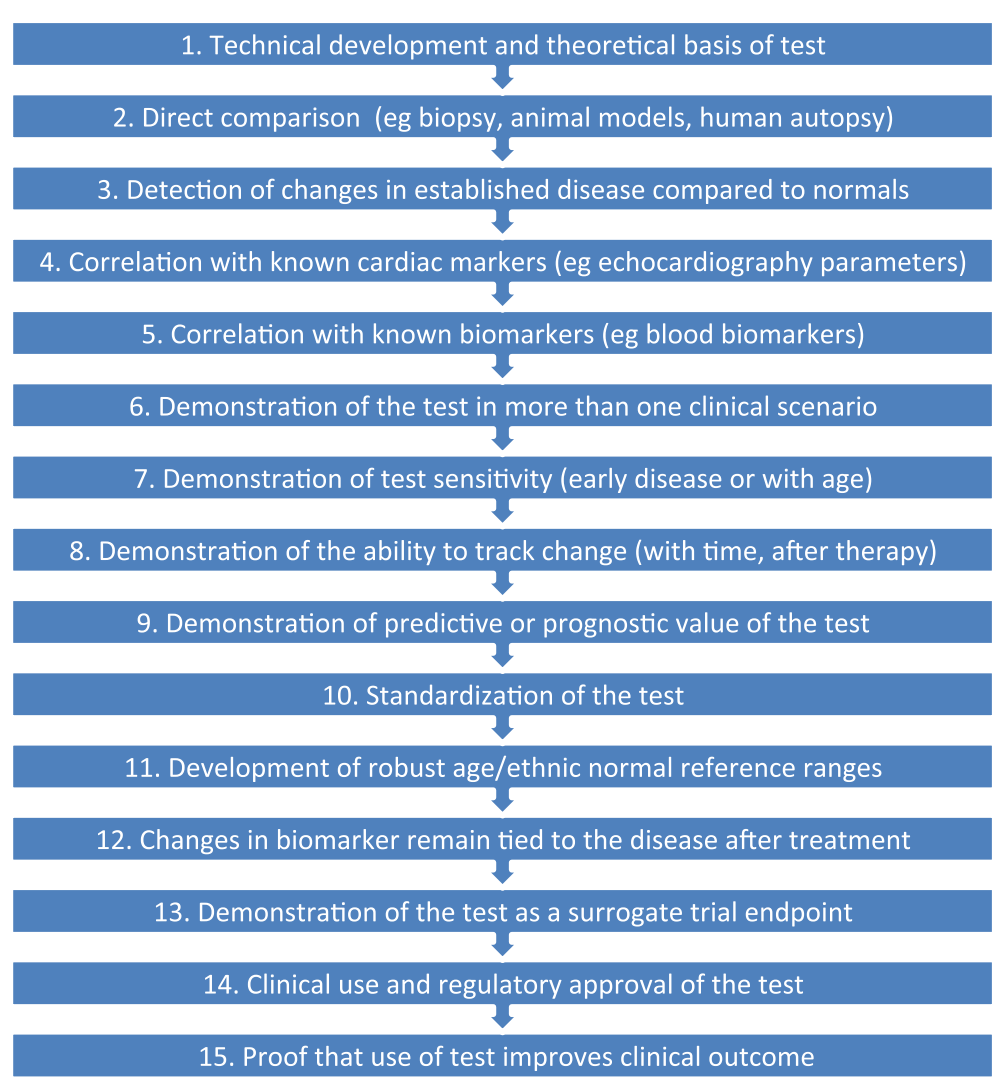

Fig. 4 Roadmap for developing biomarkers derived from parametric mapping 
Non-contrast protocols will be useful for patients with a need for repeated CMR or with kidney failure.

Eventually, data on accuracy and prognosis will have to be followed by trials on the impact on patient outcomes. It is likely that relaxation times and ECV may achieve the status of biomarkers (Fig. 4), which allow for defining the current status of the myocardium [192].

\section{Conclusion}

CMR mapping of T1, T2, T2* and ECV provides quantitative information on changes of magnetic tissue properties, which reflect alterations of myocardial tissue composition. CMR mapping methodology has left behind the early stages of principal implementation and validation, and robust techniques are available for commercial CMR systems. Iron overload, amyloidosis, Anderson-Fabry, and myocarditis are clinical scenarios where cardiac mapping provides unique information and should be applied to guide clinical care. Due to its additional diagnostic and prognostic value in the assessment of diffuse myocardial disease, parametric mapping should be considered in the diagnostic evaluation of all patients with heart failure.

This document provides recommendations related to both clinical indications and practical implementation, and summarizes the underlying rationale. Building on the 2013 Consensus statement on myocardial T1 mapping and extracellular volume quantification [2], which primarily provided guidance on technical aspects, the clinical focus of this document reflects the growing body of evidence regarding the clinical utility of this maturing field. Where they overlap, the recommendations of this document are in agreement with those of the previous consensus statement with the two exceptions that a waiting time of $10 \mathrm{~min}$ after contrast application for post-contrast T1 mapping is now regarded sufficient (formerly 15 min; see T1 mapping/ ECV) and that ECV should be given as a percentage (see Terminology).

CMR parametric mapping has seen many innovations over the last decade and continuous to attract interest from both basic and clinical researchers. Thus, any consensus document can only attempt to reflect the state of evidence at a single point in time, and will invariably start to be incomplete by the time of publication. Nevertheless, this document is meant to give guidance for CMR clinicians who would like to provide state-of-the-art tissue characterization for patients with myocardial disease.

\section{Abbreviations}

3CH: 3-chamber view; 4CH: 4-chamber view; AIR: Arrhythmia-insensitiverapid; AL: Immunoglobulin light-chain derived amyloid; ANGIE: Accelerated and navigator-gated look-locker imaging for cardiac T1 estimation; ARVD: Arrhythmogenic right-ventricular dysplasia; ATTR: Transthyretin amyloid; B0: Constant magnetic field; B1: Radio frequency field; bSSFP: Balanced steady-state free precession; CMR: Cardiovascular magnetic resonance; ECG: Electrocardiogram; ECV: Extracellular volume fraction;
Gd-DTPA: Gadolinium diethylenetriamine penta-acetic acid; GraSE: Gradientspin-echo; GRE: Gradient echo; ICD: Implanted cardioverter defibrillator; LGE: Late gadolinium enhancement; LV: Left ventricle; LVEF: Left-ventricular ejection fraction; MOLLI: Modified Look-Locker inversion recovery; MT: Magnetization transfer; ROI: Region of interest; RV: Right ventricle; SAPPHIRE: Saturation pulse prepared heart-rate-independent inversion recovery; SAP-T1: Short acquisition period T1 mapping; SASHA: Saturation recovery single-shot acquisition; SAX: Short-axis orientation; ShMOLLI: Shortened modified Look-Locker inversion recovery; STIR: Short inversion time inversion recovery; STONE: Slice-interleaved T1 mapping; TSE: Turbo spin-echo

\section{Acknowledgements}

This document was carefully reviewed by the following individuals:

\begin{tabular}{|c|c|c|}
\hline Name & Conflicts of interest & Email address \\
\hline Andrew Arai & $\begin{array}{l}\text { Siemens - Research } \\
\text { Agreements (US } \\
\text { Government } \\
\text { Cooperative Research } \\
\text { and Development } \\
\text { Agreement) } \\
\text { Bayer - Clinical Trial } \\
\text { Agreement } \\
\text { (US Government } \\
\text { Cooperative Research } \\
\text { and Development } \\
\text { Agreement) }\end{array}$ & araia@nih.gov \\
\hline Colin Berry & $\begin{array}{l}\text { The University of } \\
\text { Glasgow (employer of } \\
\text { C.B) holds a research } \\
\text { agreement with } \\
\text { Siemens Healthcare }\end{array}$ & colin.berry@glasgow.ac.uk \\
\hline $\begin{array}{l}\text { David } \\
\text { Bluemke }\end{array}$ & $\begin{array}{l}\text { Siemens: research } \\
\text { agreement and speaker } \\
\text { Philips: research } \\
\text { agreement }\end{array}$ & dbluemke@rsna.org \\
\hline $\begin{array}{l}\text { Chiara } \\
\text { Bucciarelli- } \\
\text { Ducci(for } \\
\text { EACVI) }\end{array}$ & $\begin{array}{l}\text { - CBD is supported by } \\
\text { the NIHR Biomedical } \\
\text { Centre at the University } \\
\text { Hospitals Bristol NHS } \\
\text { Foundation Trust and } \\
\text { the University of Bristol. } \\
\text { The views expressed in } \\
\text { this publication are } \\
\text { those of the author(s) } \\
\text { and not necessarily } \\
\text { those of the NHS, the } \\
\text { National Institute for } \\
\text { Health Research or the } \\
\text { Department of Health. } \\
\text { - CBD is a consultant } \\
\text { for Circle Cardiovascular } \\
\text { Imaging (Calgary, } \\
\text { Canada). }\end{array}$ & $\begin{array}{l}\text { c.bucciarelli- } \\
\text { ducci@bristol.ac.uk }\end{array}$ \\
\hline
\end{tabular}

Kelvin Chow $\quad \mathrm{KC}$ is a full-time employee of Siemens Healthcare. KC

kc3nu@hscmail.mcc.virginia.edu holds patent applications on the use of variable flip angle ramping for bSSFP quantitative magnetization prepared imaging (US Patent 2015/016,0320 A1) and on the use of high-contrast imaging for improving image 
(Continued)

\begin{tabular}{|c|c|c|}
\hline Name & Conflicts of interest & Email address \\
\hline & $\begin{array}{l}\text { registration of free-breathing } \\
\text { data (US } \\
\text { Patent 2017/007,6449 A1) }\end{array}$ & \\
\hline $\begin{array}{l}\text { David } \\
\text { Higgins }\end{array}$ & Employee of Philips & david.higgins@philips.com \\
\hline $\begin{array}{l}\text { Michael } \\
\text { Jerosch- } \\
\text { Herold }\end{array}$ & $\begin{array}{l}\text { I have no conflicts to } \\
\text { disclose }\end{array}$ & $\begin{array}{l}\text { mjerosch- } \\
\text { herold@bwh.harvard.edu }\end{array}$ \\
\hline $\begin{array}{l}\text { Tim } \\
\text { Leiner(for } \\
\text { the } \\
\text { Publications } \\
\text { Committee of } \\
\text { SCMR) }\end{array}$ & $\begin{array}{l}\text { I have no conflict of } \\
\text { interest in relation } \\
\text { to the content of this } \\
\text { consensus document }\end{array}$ & t.leiner@umcutrecht.nl \\
\hline $\begin{array}{l}\text { Stefan } \\
\text { Neubauer }\end{array}$ & No relevant disclosures & stefan.neubauer@cardiov.ox.ac.uk \\
\hline $\begin{array}{l}\text { Dudley } \\
\text { Pennell }\end{array}$ & $\begin{array}{l}\text { Research support } \\
\text { from Siemens, } \\
\text { and } \\
\text { Director \& } \\
\text { Shareholder } \\
\text { in CVIS (T2* } \\
\text { analysis software) }\end{array}$ & dj.pennell@rbht.nhs.uk \\
\hline $\begin{array}{l}\text { Subha } \\
\text { Raman }\end{array}$ & $\begin{array}{l}\text { Institutional } \\
\text { research support } \\
\text { from Siemens }\end{array}$ & raman.1@osu.edu \\
\hline $\begin{array}{l}\text { Jeanette } \\
\text { Schulz- } \\
\text { Menger }\end{array}$ & $\begin{array}{l}\text { no conflict of } \\
\text { interest in relation } \\
\text { to the content of } \\
\text { this consensus } \\
\text { document }\end{array}$ & $\begin{array}{l}\text { jeanette.schulz- } \\
\text { menger@charite.de }\end{array}$ \\
\hline Glenn Slavin & $\begin{array}{l}\text { Employment by } \\
\text { GE Healthcare }\end{array}$ & glenn.slavin@ge.com \\
\hline
\end{tabular}

Ethical approval and consent to participate

Not applicable.

\section{Funding}

Not applicable.

\section{Availability of data and materials}

Not applicable.

\section{Authors' contributions}

DRM, JCM, VF, TH, PK, JM, RN, ES, MU, RBVH, and MGF convened in London hosted by JCM, wrote sections and edited the manuscript collated by DRM. LGW, MS, AJT, and RT wrote sections and edited the manuscript. All authors read and approved the final manuscript.

\section{Authors' information}

Not applicable.

\section{Consent for publication}

All authors have read and approved the final version of this manuscript.

\section{Competing interests}

The authors declare that they have no competing interests.

\section{Publisher's Note}

Springer Nature remains neutral with regard to jurisdictional claims in published maps and institutional affiliations.

\section{Author details}

'Department of Internal Medicine and Cardiology, Deutsches Herzzentrum Berlin, Berlin, Germany. ${ }^{2}$ Department of Internal Medicine and Cardiology, Charité Universitätsmedizin Berlin, Augustenburger Platz 1, 13353 Berlin, Germany. ${ }^{3}$ DZHK (German Centre for Cardiovascular Research), partner site Berlin, Augustenburger Platz 1, 13353 Berlin, Germany. ${ }^{4}$ University College London and Barts Heart Centre, London, UK. ${ }^{5}$ Oxford Centre for Clinical Magnetic Resonance Research, Division of Cardiovascular Medicine, Radcliffe Department of Medicine, University of Oxford, Oxford, UK. ${ }^{6}$ Division of Cardiology in the Department of Pediatrics, The Hospital for Sick Children, University of Toronto, Toronto, ON, Canada. ${ }^{7}$ Cardiovascular Science Research Centre, St George's, University of London, London, UK. ${ }^{8}$ National Institutes of Health, Bethesda, MD, USA. ${ }^{9}$ Department of Internal Medicine II, Division of Cardiology, Vienna, Austria. ${ }^{10}$ Department of Medicine (Cardiovascular Division), Beth Israel Deaconess Medical Center, Harvard Medical School, Boston, USA. " Departments of Medicine Cardiology Division, Radiology and Medical Imaging, and Biomedical Engineering, University of Virginia Health System, Charlottesville, VA, USA. ${ }^{12}$ Department of Medicine, University of Pittsburgh School of Medicine, Pittsburgh, PA, USA. ${ }^{13}$ UPMC Cardiovascular Magnetic Resonance Center, Heart and Vascular Institute, Pittsburgh, PA, USA. ${ }^{14} \mathrm{Clinical}$ and Translational Science Institute, University of Pittsburgh, Pittsburgh, PA, USA. ${ }^{15}$ The Alfred Hospital, Baker Heart and Diabetes Institute, Melbourne, Australia. ${ }^{16}$ Department of Biomedical Engineering, University of Alberta, Edmonton, Canada. ${ }^{17}$ Department of Clinical Physiology, Karolinska Institutet, Karolinska University Hospital, Stockholm, Sweden. ${ }^{18}$ Department of Radiology, Lausanne University Hospital (CHUV) and Lausanne University (UNIL), Lausanne, Switzerland. ${ }^{19}$ Departments of Medicine and Diagnostic Radiology, McGill University, Montréal, Québec, Canada. ${ }^{20}$ Department of Medicine, Heidelberg University, Heidelberg, Germany. ${ }^{21}$ Département de radiologie, Université de Montréal, Montréal, Québec, Canada. ${ }^{22}$ Departments of Cardiac Sciences and Radiology, University of Calgary, Calgary, Canada.

Received: 8 July 2017 Accepted: 25 September 2017

Published online: 09 October 2017

\section{References}

1. Modell B, Khan M, Darlison M, Westwood MA, Ingram D, Pennell DJ. Improved survival of thalassaemia major in the UK and relation to $\mathrm{T}^{*}$ cardiovascular magnetic resonance. J Cardiovasc Magn Reson. 2008;10:42.

2. Moon JC, Messroghli DR, Kellman P, Piechnik SK, Robson MD, Ugander M, et al. Myocardial T1 mapping and extracellular volume quantification: a Society for Cardiovascular Magnetic Resonance (SCMR) and CMR Working Group of the European Society of Cardiology consensus statement. J Cardiovasc Magn Reson. 2013;15:92.

3. Messroghli DR, Radjenovic A, Kozerke S, Higgins DM, Sivananthan MU, Ridgway JP. Modified Look-Locker inversion recovery (MOLLI) for highresolution T1 mapping of the heart. Magn Reson Med. 2004;52:141-6.

4. Messroghli DR, Plein S, Higgins DM, Walters K, Jones TR, Ridgway JP, et al. Human myocardium: single-breath-hold MR T1 mapping with high spatial resolution-reproducibility study. Radiology. 2006;238:1004-12.

5. Messroghli DR, Greiser A, Fröhlich M, Dietz R, Schulz-Menger J. Optimization and validation of a fully-integrated pulse sequence for modified look-locker inversion-recovery (MOLLI) T1 mapping of the heart. J Magn Reson Imaging. 2007;26:1081-6.

6. Piechnik SK, Ferreira VM, Dall'Armellina E, Cochlin LE, Greiser A, Neubauer S, et al. Shortened Modified Look-Locker Inversion recovery (ShMOLLI) for clinical myocardial T1-mapping at 1.5 and 3 T within a 9 heartbeat breathhold. J Cardiovasc Magn Reson. 2010;12:69.

7. Mehta BB, Chen X, Bilchick KC, Salerno M, Epstein FH. Accelerated and navigator-gated look-locker imaging for cardiac t1 estimation (ANGIE): Development and application to T1 mapping of the right ventricle. Magn Reson Med. 2015;73(1):150-60.

8. Weingärtner S, Roujol S, Akçakaya M, Basha TA, Nezafat R. Free-breathing multislice native myocardial T1 mapping using the slice-interleaved T1 (STONE) sequence. Magn Reson Med. 2014; 
9. Fitts M, Breton E, Kholmovski EG, Dosdall DJ, Vijayakumar S, Hong KP, et al. Arrhythmia insensitive rapid cardiac T1 mapping pulse sequence. Magn Reson Med. 2013;70:1274-82

10. Chow K, Flewitt JA, Green JD, Pagano JJ, Friedrich MG, Thompson RB. Saturation recovery single-shot acquisition (SASHA) for myocardial T1 mapping. Magn Reson Med. 2014;71:2082-95.

11. Higgins DM, Ridgway JP, Radjenovic A, Sivananthan UM, Smith MA. T1 measurement using a short acquisition period for quantitative cardiac applications. Med Phys. 2005;32:1738-46.

12. Weingärtner S, Akçakaya M, Basha T, Kissinger KV, Goddu B, Berg S, et al. Combined saturation/inversion recovery sequences for improved evaluation of scar and diffuse fibrosis in patients with arrhythmia or heart rate variability. Magn Reson Med. 2014;71:1024-34.

13. Look DC, Locker DR. Time saving in measurement of NMR and EPR relaxation times. Rev Sci Instrum. 1970;41:250-1.

14. Messroghli DR, Walters K, Plein S, Sparrow P, Friedrich MG, Ridgway JP, et al. Myocardial T1 mapping: application to patients with acute and chronic myocardial infarction. Magn Reson Med. 2007;58:34-40.

15. Robson MD, Piechnik SK, Tunnicliffe EM, Neubauer S. T1 measurements in the human myocardium: The effects of magnetization transfer on the SASHA and MOLLI sequences. Magn Reson Med. 2013; Epub ahead of print

16. Kellman P, Hansen MS. T1-mapping in the heart: accuracy and precision. J Cardiovasc Magn Reson. 2014;16:2.

17. Kellman P, Herzka DA, Arai AE, Hansen MS. Influence of Off-resonance in myocardial T1-mapping using SSFP based MOLLI method. J CardiovasC Magn Reson. 2013;15:63

18. Kellman P, Herzka DA, Hansen MS. Adiabatic inversion pulses for myocardial T1 mapping. Magn Reson Med. 2014;71:1428-34.

19. Roujol S, Weingärtner S, Foppa M, Chow K, Kawaji K, Ngo LH, et al. Accuracy, Precision, and Reproducibility of Four T1 Mapping Sequences: A Head- to-Head Comparison of MOLLI, ShMOLLI, SASHA, and SAPPHIRE. Radiology. 2014;272:683-9.

20. Bhuva AN, Nordin S, Bulluck H, Treibel TA, Abdel-Gadir A, Rosmini S, et al. Reproducibility of native T1 mapping using ShMOLLI and MOLLI - implications for sample size calculation. J Cardiovasc Magn Reson. 2016;18:P2.

21. Kellman P, Arai AE, Xue H. T1 and extracellular volume mapping in the heart: estimation of error maps and the influence of noise on precision. J Cardiovasc Magn Reson. 2013;15

22. Kellman P, Wilson JR, Xue H, Ugander M, Arai AE. Extracellular volume fraction mapping in the myocardium, part 1: evaluation of an automated method. J Cardiovasc Magn Reson. 2012;14:63.

23. Marty B, Vignaud A, Greiser A, Robert B, de Sousa PL, Carlier PG. BLOCH equations-based reconstruction of myocardium $t 1$ maps from modified look-locker inversion recovery sequence. PLoS One. 2015;10:e0126766.

24. Shao J, Rapacchi S, Nguyen K-L, Hu P. Myocardial T1 mapping at 3.0 tesla using an inversion recovery spoiled gradient echo readout and Bloch equation simulation with slice profile correction (BLESSPC) T1 estimation algorithm. J Magn Reson Imaging. 2016;43:414-25.

25. Shao J, Nguyen K-L, Natsuaki Y, Spottiswoode B, Hu P. Instantaneous signal loss simulation (InSiL): an improved algorithm for myocardial T1 mapping using the MOLLI sequence. J Magn Reson Imaging. 2015;41:721-9.

26. Chow K, Yang Y, Shaw P, Kramer CM, Salerno M. Robust free-breathing SASHA T1 mapping with high-contrast image registration. J CardiovasC Magn Reson. 2016;18:47.

27. Ferreira VM, Wijesurendra RS, Liu A, Greiser A, Casadei B, Robson MD, et al. Systolic ShMOLLI myocardial T1-mapping for improved robustness to partial-volume effects and applications in tachyarrhythmias. J Cardiovasc Magn Reson. 2015;17:77.

28. Zhao L, Li S, Ma X, Greiser A, Zhang T, An J, et al. Systolic MOLLI T1 mapping with heart-rate-dependent pulse sequence sampling scheme is feasible in patients with atrial fibrillation. J Cardiovasc Magn Reson. 2016;18:13.

29. Miller CA, Naish JH, Bishop P, Coutts G, Clark D, Zhao S, et al. Comprehensive validation of cardiovascular magnetic resonance techniques for the assessment of myocardial extracellular volume. Circ Cardiovasc Imaging. 2013;6:373-83.

30. Flett AS, Sado DM, Quarta G, Mirabel M, Pellerin D, Herrey AS, et al. Diffuse myocardial fibrosis in severe aortic stenosis: an equilibrium contrast cardiovascular magnetic resonance study. Eur Hear J Cardiovasc Imaging. 2012;13:819-26.
31. Messroghli DR, Nordmeyer S, Dietrich T, Dirsch O, Kaschina E, Savvatis K, et al. Assessment of diffuse myocardial fibrosis in rats using small animal LookLocker inversion recovery (SALLI) T1 mapping. Circ Cardiovasc Imaging. 2011:4:636-40.

32. Treibel TA, Fontana M, Maestrini V, Castelletti S, Rosmini S, Simpson J, et al. Automatic measurement of the myocardial interstitium: synthetic extracellular volume quantification without hematocrit sampling. JACC Cardiovasc Imaging. 2016;9:54-63.

33. Biesbroek PS, Amier RP, Teunissen PFA, Hofman MBM, Robbers LFHJ, van de Ven PM, et al. Changes in remote myocardial tissue after acute myocardial infarction and its relation to cardiac remodeling: A CMR T1 mapping study. PLoS One. 2017;e0180115:12.

34. Xue H, Shah S, Greiser A, Guetter C, Littmann A, Jolly MP, et al. Motion correction for myocardial T1 mapping using image registration with synthetic image estimation. Magn Reson Med. 2012;67 https://doi.org/10. 1002/mrm.23153.

35. Roujol S, Foppa M, Weingärtner S, Manning WJ, Nezafat R. Adaptive registration of varying contrast-weighted images for improved tissue characterization (ARCTIC): Application to T1 mapping. Magn Reson Med. 2014;0:1-14

36. Kellman P, Arai AE, Xue H. T1 and extracellular volume mapping in the heart: estimation of error maps and the influence of noise on precision. J Cardiovasc Magn Reson. 2013;15:56.

37. Hosch W, Bock M, Libicher M, Ley S, Hegenbart U, Dengler TJ, et al. MR-relaxometry of myocardial tissue: significant elevation of T1 and $\mathrm{T} 2$ relaxation times in cardiac amyloidosis. Investig Radiol. 2007;42:636-42.

38. McNamara MT, Higgins CB, Schechtmann N, Botvinick E, Lipton MJ, Chatterjee $\mathrm{K}$, et al. Detection and characterization of acute myocardial infarction in man with use of gated magnetic resonance. Circulation. 1985; 71:717-24.

39. Giri S, Chung Y-C, Merchant A, Mihai G, Rajagopalan S, Raman SV, et al. T2 quantification for improved detection of myocardial edema. J Cardiovasc Magn Reson. 2009;11:56

40. Foltz WD, Al-Kwifi O, Sussman MS, Stainsby JA, Wright G. a. Optimized spiral imaging for measurement of myocardial T2 relaxation. Magn Reson Med. 2003;49:1089-97.

41. Huang T-Y, Liu Y-J, Stemmer A, Poncelet BP. T2 measurement of the human myocardium using a T2-prepared transient-state TrueFISP sequence. Magn Reson Med. 2007:57:960-6.

42. van Heeswijk RB, Feliciano H, Bongard C, Bonanno G, Coppo S, Lauriers N, et al. Free-breathing $3 \mathrm{~T}$ magnetic resonance T2-mapping of the heart. JACC Cardiovasc Imaging. 2012;5:1231-9.

43. Sprinkart AM, Luetkens JA, Träber F, Doerner J, Gieseke J, Schnackenburg B, et al. Gradient Spin Echo (GraSE) imaging for fast myocardial T2 mapping. J Cardiovasc Magn Reson. 2015;17:12.

44. Akçakaya M, Basha TA, Weingärtner S, Roujol S, Berg S, Nezafat R. Improved quantitative myocardial T2 mapping: Impact of the fitting model. Magn Reson Med. 2015;74(1):93-105.

45. Roujol S, Basha TA, Weingärtner S, Akçakaya M, Berg S, Manning WJ, et al. Impact of motion correction on reproducibility and spatial variability of quantitative myocardial T2 mapping. J Cardiovasc Magn Reson. 2015;17:46.

46. Bellm S, Basha TA, Shah R V, Murthy VL, Liew C, Tang M, et al. Reproducibility of myocardial $\mathrm{T} 1$ and $\mathrm{T} 2$ relaxation time measurement using slice-interleaved T1 and T2 mapping sequences. J Magn Reson Imaging. 2016;44(5):1159-167.

47. Baeßler B, Schaarschmidt F, Stehning C, Schnackenburg B, Giolda A, Maintz D, et al. Reproducibility of three different cardiac T2 -mapping sequences at 1.5T. J Magn Reson Imaging. 2016;44(5):1168-178.

48. von Knobelsdorff-Brenkenhoff F, Prothmann M, Dieringer MA, Wassmuth R, Greiser A, Schwenke C, et al. Myocardial T1 and T2 mapping at 3 T: reference values, influencing factors and implications. J Cardiovasc Magn Reson. 2013;15:53.

49. Wassmuth R, Prothmann M, Utz W, Dieringer M, von Knobelsdorff-Brenkenhoff F, Greiser A, et al. Variability and homogeneity of cardiovascular magnetic resonance myocardial T2-mapping in volunteers compared to patients with edema. J Cardiovasc Magn Reson. 2013;15:27.

50. McAlindon EJ, Pufulete M, Harris JM, Lawton CB, Moon JC, Manghat N, et al. Measurement of myocardium at risk with cardiovascular MR: comparison of techniques for edema imaging. Radiology. 2015;275:61-70. 
51. Bano W, Feliciano H, Coristine AJ, Stuber M, van Heeswijk RB. On the accuracy and precision of cardiac magnetic resonance T2 mapping: A highresolution radial study using adiabatic $\mathrm{T} 2$ preparation at $3 \mathrm{~T}$. Magn Reson Med. 2016;

52. Giri S, Shah S, Xue H, Chung Y-C, Pennell ML, Guehring J, et al. Myocardial T2 mapping with respiratory navigator and automatic nonrigid motion correction. Magn Reson Med. 2012;68:1570-8.

53. van Heeswijk RB, Piccini D, Feliciano H, Hullin R, Schwitter J, Stuber M. Selfnavigated isotropic three-dimensional cardiac T2 mapping. Magn Reson Med. 2015;73:1549-54.

54. Ding $H$, Fernandez-de-Manuel L, Schär M, Schuleri $\mathrm{KH}$, Halperin $H$, He L, et al. Three-dimensional whole-heart T2 mapping at 3T. Magn Reson Med. 2015:74:803-16

55. Basha TA, Bellm S, Roujol S, Kato S, Nezafat R. Free-breathing sliceinterleaved myocardial T2 mapping with slice-selective T2 magnetization preparation. Magn Reson Med. 2016;76:555-65.

56. Chavhan GB, Babyn PS, Thomas B, Shroff MM, Haacke EM. Principles, techniques, and applications of T2*-based MR imaging and its special applications. Radiographics. 29:1433-49.

57. Brittenham GM. Iron-chelating therapy for transfusional iron overload. N Engl J Med. 2011;364:146-56.

58. Westwood M, Anderson LJ, Firmin DN, Gatehouse PD, Charrier CC, Wonke B, et al. A single breath-hold multiecho $\mathrm{T}^{*}$ cardiovascular magnetic resonance technique for diagnosis of myocardial iron overload. J Magn Reson Imaging. 2003;18:33-9.

59. He T, Gatehouse PD, Kirk P, Tanner MA, Smith GC, Keegan J, et al. Blackblood T2* technique for myocardial iron measurement in thalassemia. J Magn Reson Imaging. 2007;25:1205-9.

60. Kirk P, He T, Anderson L, Roughton M, Tanner MA, Lam WWM, et al. International reproducibility of single breathhold T2* MR for cardiac and liver iron assessment among five thalassemia centers. J Magn Reson Imaging. 2010:32:315-9.

61. Smith GC, Carpenter JP, He T, Alam MH, Firmin DN, Pennell DJ. Value of black blood T2* cardiovascular magnetic resonance. J Cardiovasc Magn Reson. 2011;13:21.

62. Pennell DJ, Udelson JE, Arai AE, Bozkurt B, Cohen AR, Galanello R, et al. Cardiovascular function and treatment in $\beta$-thalassemia major: a consensus statement from the American Heart Association. Circulation. 2013;128:281-308

63. Baksi AJ, Pennell DJ. T2* imaging of the heart: methods, applications, and outcomes. Top Magn Reson Imaging. 2014;23:13-20.

64. Ferreira VM, Piechnik SK, Dall'Armellina E, Karamitsos TD, Francis JM, Choudhury RP, et al. Non-contrast T1-mapping detects acute myocardial edema with high diagnostic accuracy: a comparison to T2-weighted cardiovascular magnetic resonance. J Cardiovasc Magn Reson. 2012;14:42.

65. Peller M, Kurze V, Loeffler R, Pahernik S, Dellian M, Goetz AE, et al. Hyperthermia induces T1 relaxation and blood flow changes in tumors. A MRI thermometry study in vivo. Magn Reson Imaging. 2003;21:545-51.

66. Dalager-Pedersen S, Falk E, Ringgaard S, Kristensen IB, Pedersen EM. Effects of temperature and histopathologic preparation on the size and morphology of atherosclerotic carotid arteries as imaged by MRI. J Magn Reson Imaging. 1999;10:876-85

67. Captur G, Gatehouse P, Keenan KE, Heslinga FG, Bruehl R, Prothmann M, et al. A medical device-grade T1 and ECV phantom for global T1 mapping quality assurance - the T1 Mapping and ECV standardization in cardiovascular magnetic resonance (T1MES) program. J Cardiovasc Magn Reson. 2016:18:58.

68. Kirk P, Smith GC, Roughton M, He T, Pennell DJ. Myocardial T2* is not affected by ageing, myocardial fibrosis, or impaired left ventricular function. J Magn Reson Imaging. 2010;32:1095-8.

69. Liu C-Y, Liu Y-C, Wu C, Armstrong A, Volpe GJ, van der Geest RJ, et al. Evaluation of age-related interstitial myocardial fibrosis with cardiac magnetic resonance contrast-enhanced T1 mapping: MESA (Multi-Ethnic Study of Atherosclerosis). J Am Coll Cardiol. 2013;62:1280-7.

70. Piechnik SK, Ferreira VM, Lewandowski AJ, Ntusi N. a B, Banerjee R, Holloway $C$, et al. Normal variation of magnetic resonance $T 1$ relaxation times in the human population at $1.5 \mathrm{~T}$ using ShMOLLI. J CardiovasC Magn Reson. 2013;15:13.

71. Bönner F, Janzarik N, Jacoby C, Spieker M, Schnackenburg B, Range F, et al. Myocardial T2 mapping reveals age- and sex-related differences in volunteers. J Cardiovasc Magn Reson. 2015;17:9.
72. Petersen SE, Matthews PM, Francis JM, Robson MD, Zemrak F, Boubertakh R, et al. UK Biobank's cardiovascular magnetic resonance protocol. J Cardiovasc Magn Reson. 2015;18:8.

73. Williams ES, Kaplan Jl, Thatcher F, Zimmerman G, Knoebel SB. Prolongation of proton spin lattice relaxation times in regionally ischemic tissue from dog hearts. J Nucl Med. 1980;21:449-53.

74. Fernández-Jiménez R, Sánchez-González J, Aguero J, Del Trigo M, GalánArriola C, Fuster V, et al. Fast T2 gradient-spin-echo (T2-GraSE) mapping for myocardial edema quantification: first in vivo validation in a porcine model of ischemia/reperfusion. J Cardiovasc Magn Reson. 2015;17:92.

75. Ugander M, Bagi PS, Oki AJ, Chen B, Hsu L-Y, Aletras AH, et al. Myocardial edema as detected by pre-contrast T1 and T2 CMR delineates area at risk associated with acute myocardial infarction. JACC CardiovasC Imaging. 2012;5:596-603.

76. Goldfarb JW, Arnold S, Han J. Recent myocardial infarction: assessment with unenhanced T1-weighted MR imaging. Radiology. 2007;245:245-50.

77. Bulluck H, White SK, Rosmini S, Bhuva A, Treibel TA, Fontana M, et al. T1 mapping and T2 mapping at 3T for quantifying the area-at-risk in reperfused STEMI patients. J Cardiovasc Magn Reson. 2015;17:73.

78. Schelbert EB, Messroghli DR. Clinical applications of cardiac T1 mapping. Radiology. 2015;278:658-76.

79. Armellina ED, Piechnik SK, Ferreira VM, Si QL, Robson MD, Francis JM, et al. Cardiovascular magnetic resonance by non contrast T1-mapping allows assessment of severity of injury in acute myocardial infarction. 2012;1-13.

80. Carrick D, Haig C, Rauhalammi S, Ahmed N, Mordi I, McEntegart M, et al. Prognostic significance of infarct core pathology revealed by quantitative non-contrast in comparison with contrast cardiac magnetic resonance imaging in reperfused ST-elevation myocardial infarction survivors. Eur Heart J. 2016;37(13):1044-59. https://doi.org/10.1093/eurheartj/ehv372.

81. Pedersen SF, Thrysøe SA, Robich MP, Paaske WP, Ringgaard S, Bøtker HE, et al. Assessment of intramyocardial hemorrhage by T1-weighted cardiovascular magnetic resonance in reperfused acute myocardial infarction. J Cardiovasc Magn Reson. 2012;14:59.

82. Chan W, Duffy SJ, White DA, Gao X-M, Du X-J, Ellims AH, et al. Acute left ventricular remodeling following myocardial infarction: coupling of regional healing with remote extracellular matrix expansion. JACC CardiovasC Imaging. 2012;5:884-93.

83. Carrick D, Haig C, Rauhalammi S, Ahmed N, Mordi I, McEntegart M, et al. Pathophysiology of LV Remodeling in Survivors of STEMI: Inflammation, Remote Myocardium, and Prognosis. JACC CardiovasC Imaging. 2015;8:779-89.

84. Carberry J, Carrick D, Haig C, Rauhalammi SM, Ahmed N, Mordi I, et al. Remote zone extracellular volume and left ventricular remodeling in survivors of ST-elevation myocardial infarction. Hypertension. 2016; 68:385-91.

85. Zia MI, Ghugre NR, Connelly KA, Strauss BH, Sparkes JD, Dick AJ, et al. Characterizing myocardial edema and hemorrhage using quantitative T2 and $\mathrm{T}^{*}$ mapping at multiple time intervals post ST-segment elevation myocardial infarction. Circ Cardiovasc Imaging. 2012;5:566-72.

86. Higgins CB, Herfkens R, Lipton MJ, Sievers R, Sheldon P, Kaufman L, et al. Nuclear magnetic resonance imaging of acute myocardial infarction in dogs: alterations in magnetic relaxation times. Am J Cardiol. 1983;52:184-8.

87. Ferreira VM, Piechnik SK, Dall'armellina E, Karamitsos TD, Francis JM, Ntusi N, et al. T1 Mapping for the Diagnosis of Acute Myocarditis Using CMR: Comparison to T2-weighted and late gadolinium enhanced imaging. JACC Cardiovasc Imaging. 2013;2013:1048-58.

88. Thavendiranathan P, Walls M, Giri S, Verhaert D, Rajagopalan S, Moore S, et al. Improved detection of myocardial involvement in acute inflammatory cardiomyopathies using T2 mapping. Circ Cardiovasc Imaging. 2012;5:102-10.

89. Verhaert D, Thavendiranathan P, Giri S, Mihai G, Rajagopalan S, Simonetti $O P$, et al. Direct t2 quantification of myocardial edema in acute ischemic injury. JACC Cardiovasc Imaging. 2011;4:269-78.

90. Ferreira VM, Piechnik SK, Dall'Armellina E, Karamitsos TD, Francis JM, Ntusi N, et al. Native T1-mapping detects the location, extent and patterns of acute myocarditis without the need for gadolinium contrast agents. J Cardiovasc Magn Reson. 2014;16:36

91. Lurz P, Luecke C, Eitel I, Föhrenbach F, Frank C, Grothoff M, et al. Comprehensive cardiac magnetic resonance imaging in patients with suspected myocarditis: The MyoRacer-Trial. J Am Coll Cardiol. 2016;67:1800-11. 
92. Ntusi NA, Piechnik SK, Francis JM, Ferreira VM, Rai AB, Matthews PM, et al. Subclinical myocardial inflammation and diffuse fibrosis are common in systemic sclerosis-a clinical study using myocardial T1-mapping and extracellular volume quantification. J Cardiovasc Magn Reson. 2014;16: 21.

93. Ntusi NAB, Piechnik SK, Francis JM, Ferreira VM, Matthews PM, Robson MD, et al. Diffuse myocardial fibrosis and inflammation in rheumatoid arthritis: insights from CMR T1 mapping. JACC Cardiovasc Imaging. 2015:8:526-36.

94. Puntmann VO, D'Cruz D, Smith Z, Pastor A, Choong P, Voigt T, et al. Native myocardial T1 mapping by cardiovascular magnetic resonance imaging in subclinical cardiomyopathy in patients with systemic lupus erythematosus. Circ Cardiovasc Imaging. 2013;6:295-301.

95. Ferreira VM, Marcelino M, Piechnik SK, Marini C, Karamitsos TD, Ntusi NAB, et al. Pheochromocytoma is characterized by catecholamine-mediated myocarditis, focal and diffuse myocardial fibrosis, and myocardial dysfunction. J Am Coll Cardiol. 2016;67:2364-74.

96. Ntusi N, O'Dwyer E, Dorrell L, Wainwright E, Piechnik S, Clutton G, et al. HIV1-related cardiovascular disease is associated with chronic inflammation, frequent pericardial effusions, and probable myocardial edema. Circ Cardiovasc Imaging. 2016;e004430:9.

97. Crouser ED, Ono C, Tran T, He X, Raman SV. Improved detection of cardiac sarcoidosis using magnetic resonance with myocardial T2 mapping. Am J Respir Crit Care Med. 2014;189:109-12.

98. Usman AA, Taimen K, Wasielewski M, McDonald J, Shah S, Giri S, et al. Cardiac magnetic resonance T2 mapping in the monitoring and follow-up of acute cardiac transplant rejection: A pilot study. Circ Cardiovasc Imaging. 2012:5:782-90.

99. Mordi I, Carrick D, Bezerra H, Tzemos N. T1 and T2 mapping for early diagnosis of dilated non-ischaemic cardiomyopathy in middle-aged patients and differentiation from normal physiological adaptation. Eur Heart J Cardiovasc Imaging. 2015;

100. Dweck MR, Boon NA, Newby DE. Calcific aortic stenosis: A disease of the valve and the myocardium. J Am Coll Cardiol. 2012;60:1854-63.

101. Cioffi G, Faggiano P, Vizzardi E, Tarantini L, Cramariuc D, Gerdts E, et al. Prognostic effect of inappropriately high left ventricular mass in asymptomatic severe aortic stenosis. Heart. 2011;97:301-7.

102. Lee S-P, Lee W, Lee JM, Park E-A, Kim H-K, Kim Y-J, et al. Assessment of diffuse myocardial fibrosis by using MR imaging in asymptomatic patients with aortic stenosis. Radiology. 2015;274:359-69.

103. White SK, Sado DM, Fontana M, Banypersad SM, Maestrini V, Flett AS, et al. T1 mapping for myocardial extracellular volume measurement by CMR: bolus only versus primed infusion technique. JACC Cardiovasc Imaging. 2013;6:955-62.

104. Mahmod M, Piechnik SK, Levelt E, Ferreira VM, Francis JM, Lewis A, et al. Adenosine stress native T1 mapping in severe aortic stenosis: evidence for a role of the intravascular compartment on myocardial T1 values. J Cardiovasc Magn Reson. 2014;16:92.

105. Treibel TA, Zemrak F, Sado DM, Banypersad SM, White SK, Maestrini V, et al. Extracellular volume quantification in isolated hypertension - changes at the detectable limits? J Cardiovasc Magn Reson. 2015;17:74.

106. Kuruvilla S, Janardhanan R, Antkowiak P, Keeley EC, Adenaw N, Brooks J, et al. Increased extracellular volume and altered mechanics are associated with LVH in hypertensive heart disease, not hypertension alone. JACC Cardiovasc Imaging. 2015;8:172-80.

107. Rodrigues JCL, Amadu AM, Dastidar AG, Szantho GV, Lyen SM, Godsave C, et al. Comprehensive characterisation of hypertensive heart disease left ventricular phenotypes. Heart. 2016;102:1671-9.

108. Hinojar R, Varma N, Child N, Goodman B, Jabbour A, Yu C-Y, et al. T1 Mapping in Discrimination of hypertrophic phenotypes: hypertensive heart disease and hypertrophic cardiomyopathy: findings from the international T1 multicenter cardiovascular magnetic resonance study. Circ Cardiovasc Imaging. 2015:8.

109. McLellan AJA, Schlaich MP, Taylor AJ, Prabhu S, Hering D, Hammond L, et al. Reverse cardiac remodeling after renal denervation: Atrial electrophysiologic and structural changes associated with blood pressure lowering. Hear Rhythm. 2016;12:982-90.

110. Karamitsos TD, Piechnik SK, Banypersad SM, Fontana M, Ntusi NB, Ferreira VM, et al. Noncontrast T1 mapping for the diagnosis of cardiac amyloidosis. JACC Cardiovasc Imaging. 2013;6:488-97.

111. Fontana M, Banypersad SM, Treibel TA, Maestrini V, Sado DM, White SK, et al. Native T1,apping in transthyretin amyloidosis. JACC Cardiovasc Imaging. 2014;7(2):157-65.
112. Banypersad SM, Sado DM, Flett AS, Gibbs SDJ, Pinney JH, Maestrini V, et al. Quantification of myocardial extracellular volume fraction in systemic AL amyloidosis: an equilibrium contrast cardiovascular magnetic resonance study. Circ Cardiovasc Imaging. 2013;6:34-9.

113. Fontana M, Pica S, Reant P, Abdel-Gadir A, Treibel TA, Banypersad SM, et al. Prognostic value of late gadolinium enhancement cardiovascular magnetic resonance in cardiac amyloidosis. Circulation. 2015;132:1570-9.

114. Fontana M, Banypersad SM, Treibel TA, Abdel-Gadir A, Maestrini V, Lane T, et al. Differential myocyte responses in patients with cardiac transthyretin amyloidosis and light-chain amyloidosis: a cardiac MR imaging study. Radiology. 2015;277:388-97.

115. Treibel TA, Fontana M, Gilbertson JA, Castelletti S, White SK, Scully PR, et al. Occult transthyretin cardiac amyloid in severe calcific aortic stenosis: prevalence and prognosis in patients undergoing surgical aortic valve replacement. Circ Cardiovasc Imaging. 2016:9.

116. Richards DB, Cookson LM, Berges AC, Barton SV, Lane T, Ritter JM, et al. Therapeutic clearance of amyloid by antibodies to serum amyloid $P$ component. N Engl J Med. 2015;373:1106-14.

117. Sado DM, White SK, Piechnik SK, Banypersad SM, Treibel T, Captur G, et al. Identification and assessment of Anderson-Fabry disease by cardiovascular magnetic resonance noncontrast myocardial T1 mapping. Circ Cardiovasc Imaging. 2013;6:392-8.

118. Thompson RB, Chow K, Khan A, Chan A, Shanks M, Paterson I, et al. T1 mapping with cardiovascular MRI is highly sensitive for fabry disease independent of hypertrophy and sex. Circ Cardiovasc Imaging. 2013;6:637-45.

119. Nordin S, Kozor R, Bulluck H, Castelletti S, Rosmini S, Abdel-Gadir A, et al. Cardiac Fabry Disease with late gadolinium enhancement is a chronic inflammatory cardiomyopathy. J Am Coll Cardiol. 2016;68:1707-8.

120. Anderson LJ, Holden S, Davis B, Prescott E, Charrier CC, Bunce NH, et al. Cardiovascular T2-star (T2*) magnetic resonance for the early diagnosis of myocardial iron overload. Eur Heart J. 2001;22:2171-9.

121. Tanner MA, He T, Westwood MA, Firmin DN, Pennell DJ. Thalassemia International Federation Heart T2* Investigators. Multi-center validation of the transferability of the magnetic resonance $T 2^{*}$ technique for the quantification of tissue iron. Haematologica. 2006;91:1388-91.

122. Westwood MA, Anderson L, Firmin DN, Gatehouse PD, Lorenz CH, Wonke $B$, et al. Interscanner reproducibility of cardiovascular magnetic resonance T2* measurements of tissue iron in thalassemia. J Magn Reson Imaging. 2003;18:616-20.

123. Kirk P, Roughton M, Porter JB, Walker JM, Tanner MA, Patel J, et al. Cardiac T2* magnetic resonance for prediction of cardiac complications in thalassemia major. Circulation. 2009;120:1961-8.

124. Tanner MA, Galanello R, Dessi C, Smith GC, Westwood MA, Agus A, et al. A randomized, placebo-controlled, double-blind trial of the effect of combined therapy with deferoxamine and deferiprone on myocardial iron in thalassemia major using cardiovascular magnetic resonance. Circulation. 2007:115:1876-84.

125. Pennell DJ, Porter JB, Piga A, Lai Y-R, El-Beshlawy A, Elalfy M, et al. Sustained improvements in myocardial T2* over 2 years in severely iron-overloaded patients with beta thalassemia major treated with deferasirox or deferoxamine. Am J Hematol. 2015:90:91-6.

126. Fernandes $J$, Sampaio EF, Fertrin K, Coelho OR, Loggetto S, Piga A, et al. Amlodipine reduces cardiac iron overload in patients with Thalassemia major: A pilot trial. Am J Med. 2013;126:834-7.

127. Feng Y, He T, Carpenter J-P, Jabbour A, Alam MH, Gatehouse PD, et al. In vivo comparison of myocardial T1 with $\mathrm{T} 2$ and $\mathrm{T} 2{ }^{*}$ in thalassaemia major. J Magn Reson Imaging. 2013;38:588-93.

128. Sado DM, Maestrini V, Piechnik SK, Banypersad SM, White SK, Flett AS, et al. Noncontrast myocardial T1 mapping using cardiovascular magnetic resonance for iron overload. J Magn Reson Imaging. 2015;41:1505-11.

129. Hanneman K, Nguyen ET, Thavendiranathan P, Ward R, Greiser A, Jolly M-P, et al. Quantification of myocardial extracellular volume fraction with cardiac MR imaging in Thalassemia Major. Radiology. 2016;279:720-30.

130. Weber KT, Brilla CG. Pathological hypertrophy and cardiac interstitium. Fibrosis and renin-angiotensin-aldosterone system. Circulation. 1991:83:1849-65.

131. Salerno M, Kramer CM. Advances in parametric mapping with CMR imaging JACC Cardiovasc Imaging. 2013;6:806-22.

132. Schelbert EB, Fonarow GC, Bonow RO, Butler J, Gheorghiade M. Therapeutic targets in heart failure: refocusing on the myocardial interstitium. J Am Coll Cardiol. 2014;63:2188-98. 
133. Flett AS, Hayward MP, Ashworth MT, Hansen MS, Taylor AM, Elliott PM, et al. Equilibrium contrast cardiovascular magnetic resonance for the measurement of diffuse myocardial fibrosis: preliminary validation in humans. Circulation. 2010;122:138-44.

134. Zeng M, Zhang N, He Y, Wen Z, Wang Z, Zhao Y, et al. Histological validation of cardiac magnetic resonance $\mathrm{T} 1$ mapping for detecting diffuse myocardial fibrosis in diabetic rabbits. J Magn Reson Imaging. 2016;44(5): $1179-185$.

135. Aus dem Siepen F, Buss SJ, Messroghli D, Andre F, Lossnitzer D, Seitz S, et al. T1 mapping in dilated cardiomyopathy with cardiac magnetic resonance: quantification of diffuse myocardial fibrosis and comparison with endomyocardial biopsy. Eur Hear J Cardiovasc Imaging. 2014;16:210-6.

136. Fontana M, White SK, Banypersad SM, Sado DM, Maestrini V, Flett AS, et al. Comparison of T1 mapping techniques for ECV quantification. Histological validation and reproducibility of ShMOLLI versus multibreath-hold T1 quantification equilibrium contrast CMR. J Cardiovasc Magn Reson. 2012;14:88.

137. de Meester de Ravenstein C, Bouzin C, Lazam S, Boulif J, Amzulescu M, Melchior J, et al. Histological validation of measurement of diffuse interstitial myocardial fibrosis by myocardial extravascular volume fraction from Modified Look-Locker imaging (MOLLI) T1 mapping at 3 T. J CardiovasC Magn Reson. 2015;17:48.

138. Inui K, Tachi M, Saito T, Kubota Y, Murai K, Kato K, et al. Superiority of the extracellular volume fraction over the myocardial $\mathrm{T} 1$ value for the assessment of myocardial fibrosis in patients with non-ischemic cardiomyopathy. Magn Reson Imaging. 2016;34:1141-5.

139. Liu A, Wijesurendra RS, Francis JM, Robson MD, Neubauer S, Piechnik SK, et al. Adenosine stress and rest T1 mapping can differentiate between ischemic, infarcted, remote, and normal,yocardium without the need for gadolinium contrast agents. JACC Cardiovasc Imaging. 2016;9:27-36.

140. Kuijpers $D$, Prakken NH, Vliegenthart $R$, van Dijkman PRM, van der Harst $P$, Oudkerk M. Caffeine intake inverts the effect of adenosine on myocardial perfusion during stress as measured by T1 mapping. Int J Cardiovasc Imaging. 2016:32:1545-53.

141. Schelbert EB, Piehler KM, Zareba KM, Moon JC, Ugander M, Messroghli DR, et al. Myocardial fibrosis quantified by extracellular volume is associated with subsequent hospitalization for heart failure, death, or both across the spectrum of ejection fraction and heart failure stage. J Am Heart Assoc. 2015;e002613:4.

142. McDiarmid AK, Swoboda PP, Erhayiem B, Ripley DP, Kidambi A, Broadbent DA, et al. Single bolus versus split dose gadolinium administration in extracellular volume calculation at 3 Tesla. J Cardiovasc Magn Reson. 2015;17:6.

143. Schelbert EB, Testa SM, Meier CG, Ceyrolles WJ, Levenson JE, Blair AJ, et al. Myocardial extravascular extracellular volume fraction measurement by gadolinium cardiovascular magnetic resonance in humans: slow infusion versus bolus. J Cardiovasc Magn Reson. 2011;13:16.

144. Kawel N, Nacif M, Zavodni A, Jones J, Liu S, Sibley CT, et al. T1 mapping of the myocardium: intra-individual assessment of the effect of field strength, cardiac cycle and variation by myocardial region. J Cardiovasc Magn Reson. 2012;14:27.

145. Chin CWL, Semple S, Malley T, White AC, Mirsadraee S, Weale PJ, et al. Optimization and comparison of myocardial T1 techniques at 3T in patients with aortic stenosis. Eur Hear J Cardiovasc Imaging. 2014;15:556-65.

146. Singh A, Horsfield MA, Bekele S, Khan JN, Greiser A, McCann GP. Myocardial $\mathrm{T} 1$ and extracellular volume fraction measurement in asymptomatic patients with aortic stenosis: reproducibility and comparison with age-matched controls. Eur Heart J Cardiovasc Imaging. 2015;16:763-70.

147. Liu S, Han J, Nacif MS, Jones J, Kawel N, Kellman P, et al. Diffuse myocardial fibrosis evaluation using cardiac magnetic resonance T1 mapping: sample size considerations for clinical trials. J Cardiovasc Magn Reson. 2012;14:90.

148. Mascherbauer J, Marzluf BA, Tufaro C, Pfaffenberger S, Graf A, Wexberg P, et al. Cardiac magnetic resonance postcontrast $\mathrm{T} 1$ time is associated with outcome in patients with heart failure and preserved ejection fraction. Circ Cardiovasc Imaging. 2013;6:1056-65.

149. Kammerlander AA, Marzluf BA, Zotter-Tufaro C, Aschauer S, Duca F, Bachmann A, et al. T1 mapping by CMR imaging: from histological validation to clinical implication. JACC Cardiovasc Imaging. 2016;9:14-23.

150. Wong TC, Piehler K, Meier CG, Testa SM, Klock AM, Aneizi AA, et al. Association between extracellular matrix expansion quantified by cardiovascular magnetic resonance and short-term mortality. Circulation. 2012;126:1206-16.

151. Wong TC, Piehler KM, Kang IA, Kadakkal A, Kellman P, Schwartzman DS, et al. Myocardial extracellular volume fraction quantified by cardiovascular magnetic resonance is increased in diabetes and associated with mortality and incident heart failure admission. Eur Heart J. 2014;35:657-64.
152. Banypersad SM, Fontana M, Maestrini V, Sado DM, Captur G, Petrie A, et al. T1 mapping and survival in systemic light-chain amyloidosis. Eur Heart J. 2015;36(4):244-51.

153. Duca F, Kammerlander AA, Tufaro C, Aschauer S, Schwaiger ML, Marzluf BA, et al. Interstitial fibrosis, functional status, and outcomes in heart failure with preserved ejection fraction: Insights from a prospective cardiac magnetic resonance imaging study. Circ Cardiovasc imaging.

154. Duca F, Zotter-Tufaro C, Kammerlander AA, Panzenböck A, Aschauer S, Dalos D, et al. Cardiac extracellular matrix is associated with adverse outcome in patients with chronic heart failure. Eur J Heart Fail. 2017;19(4): 502-11.

155. Aoki T, Fukumoto Y, Sugimura K, Oikawa M, Satoh K, Nakano M, et al. Prognostic impact of myocardial interstitial fibrosis in non-ischemic heart failure. -Comparison between preserved and reduced ejection fraction heart failure. Circ J. 2011;75:2605-13.

156. Dass S, Suttie JJ, Piechnik SK, Ferreira VM, Holloway CJ, Banerjee R, et al. Myocardial tissue characterization using magnetic resonance noncontrast T1 mapping in hypertrophic and dilated cardiomyopathy. Circ Cardiovasc Imaging. 2012;5:726-33.

157. Puntmann VO, Carr-White G, Jabbour A, Yu C-Y, Gebker R, Kelle S, et al. T1Mapping and Outcome in Nonischemic Cardiomyopathy. JACC Cardiovasc Imaging. 2016;9:40-50.

158. aus dem Siepen F, Buss SJ, Messroghli D, Andre F, Lossnitzer D, Seitz S, et al. T1 mapping in dilated cardiomyopathy with cardiac magnetic resonance: quantification of diffuse myocardial fibrosis and comparison with endomyocardial biopsy. Eur Hear J Cardiovasc Imaging. 2015;16(2):210-6.

159. Ho CY, Abbasi SA, Neilan TG, Shah RV, Chen Y, Heydari B, et al. T1 Measurements identify extracellular volume expansion in hypertrophic cardiomyopathy sarcomere mutation carriers with and without left ventricular hypertrophy. Circ Cardiovasc Imaging. 2013;6:415-22

160. Dusenbery SM, Jerosch-Herold M, Rickers C, Colan SD, Geva T, Newburger JW, et al. Myocardial extracellular remodeling is associated with ventricular diastolic dysfunction in children and young adults with congenital aortic stenosis. J Am Coll Cardiol. 2014;63:1778-85.

161. Sparrow P, Messroghli DR, Reid S, Ridgway JP, Bainbridge G, Sivananthan MU. Myocardial T1 mapping for detection of left ventricular myocardial fibrosis in chronic aortic regurgitation: pilot study. AJR Am J Roentgenol. 2006;187:W630-5.

162. Edwards NC, Moody WE, Yuan M, Weale P, Neil D, Townend J, et al. Quantification of left ventricular interstitial fibrosis in asymptomatic chronic primary degenerative mitral regurgitation world wide web at : Quantification of left ventricular interstitial fibrosis in asymptomatic chronic primary degenerative mitral reg. Circ Cardiovasc Imaging. 2014; 7:946-53

163. Kali A, Choi E-Y, Sharif B, Kim YJ, Bi X, Spottiswoode B, et al. Native T1 Mapping by 3-T CMR Imaging for Characterization of Chronic Myocardial Infarctions. JACC Cardiovasc Imaging. 2015;8:1019-30.

164. Carrick D, Haig C, Rauhalammi S, Ahmed N, Mordi I, McEntegart M, et al. Pathophysiology of LV remodelling in survivors of STEMI: inflammation, remote myocardium, and prognosis. JACC Cardiovasc Imaging. 2015;8(7): 779-89.

165. Wacker CM, Fidler F, Dueren C, Hirn S, Jakob PM, Ertl G, et al. Quantitative assessment of myocardial perfusion with a spin-labeling technique: Preliminary results in patients with coronary artery disease. J Magn Reson Imaging. 2003;18:555-60.

166. Riesenkampff E, Messroghli DR, Redington AN, Grosse-Wortmann L. Myocardial T1 mapping in pediatric and congenital heart disease. Circ Cardiovasc Imaging. 2015;8:e002504.

167. Chen C-A, Dusenbery SM, Valente AM, Powell AJ, Geva T. Myocardial ECV fraction assessed by CMR is associated with type of hemodynamic load and arrhythmia in repaired Tetralogy of Fallot. JACC Cardiovasc Imaging. 2016;9:1-10.

168. Riesenkampff E, Luining W, Seed M, Chungsomprasong P, Manlhiot C, Elders $B$, et al. Increased left ventricular myocardial extracellular volume is associated with longer cardiopulmonary bypass times, biventricular enlargement and reduced exercise tolerance in children after repair of Tetralogy of Fallot. J Cardiovasc Magn Reson. 2017;18:75.

169. Broberg CS, Huang J, Hogberg I, McLarry J, Woods P, Burchill L, et al. Diffuse LV myocardial fibrosis and its clinical associations in adults with repaired Tetralogy of Fallot. JACC Cardiovasc Imaging. 2016;9:86-7. 
170. Sarikouch S, Boethig D, Peters B, Kropf S, Dubowy K-O, Lange P, et al. Poorer right ventricular systolic function and exercise capacity in women after repair of tetralogy of fallot: a sex comparison of standard deviation scores based on sex-specific reference values in healthy control subjects. Circ Cardiovasc Imaging. 2013;6:924-33.

171. Anderson PAW, Sleeper LA, Mahony L, Colan SD, Atz AM, Breitbart RE, et al. Contemporary outcomes after the Fontan procedure: a Pediatric Heart Network multicenter study. J Am Coll Cardiol. 2008;52:85-98.

172. Plymen CM, Sado DM, Taylor AM, Bolger AP, Lambiase PD, Hughes M, et al. Diffuse myocardial fibrosis in the systemic right ventricle of patients late after Mustard or Senning surgery: an equilibrium contrast cardiovascular magnetic resonance study. Eur Heart J Cardiovasc Imaging. 2013;14:963-8.

173. Messroghli DR, Nordmeyer S, Buehrer M, Kozerke S, Dietrich T, Kaschina E, et al. Small animal Look-Locker Inversion Recovery (SALLI) for simultaneous generation of cardiac T1 maps and cine and inversion recovery - prepared images at high heart rates : initial experience. Radiology. 2011;261:258-65.

174. Soslow JH, Damon SM, Crum K, Lawson MA, Slaughter JC, Xu M, et al. Increased myocardial native T1 and extracellular volume in patients with Duchenne muscular dystrophy. J Cardiovasc Magn Reson. 2016;18:5

175. Olivieri LJ, Kellman P, McCarter RJ, Cross RR, Hansen MS, Spurney CF. Native $\mathrm{T} 1$ values identify myocardial changes and stratify disease severity in patients with Duchenne muscular dystrophy. J Cardiovasc Magn Reson. 2016;18:72.

176. Ferreira VM, Holloway CJ, Piechnik SK, Karamitsos TD, Neubauer S. Is it really fat? Ask a T1-map. Eur Heart J Cardiovasc Imaging. 2013;14:1060.

177. Reiter U, Reiter G, Asslaber M, Dacar D, Maderthaner R, Binder J, et al. Characterization of a calcified intra-cardiac pseudocyst of the mitral valve by magnetic resonance imaging including $\mathrm{T} 1$ and $\mathrm{T} 2$ mapping. BMC Cardiovasc Disord. 2014;14:11.

178. Kellman P, Bandettini WP, Mancini C, Hammer-Hansen S, Hansen MS, Arai AE. Characterization of myocardial T1-mapping bias caused by intramyocardial fat in inversion recovery and saturation recovery techniques. J Cardiovasc Magn Reson. 2015;17:33.

179. Mozes FE, Tunnicliffe EM, Pavlides M, Robson MD. Influence of fat on liver $T_{1}$ measurements using modified Look-Locker inversion recovery (MOLLI) methods at 3T. J Magn Reson Imaging. 2016:44:105-11.

180. He T, Gatehouse PD, Kirk P, Mohiaddin RH, Pennell DJ, Firmin DN. Myocardial $T^{*} 2$ measurement in iron-overloaded thalassemia: an ex vivo study to investigate optimal methods of quantification. Magn Reson Med. 2008;60:350-6.

181. Feng $Y$, He T, Feng M, Carpenter J-P, Greiser A, Xin X, et al. Improved pixelby-pixel MRI R2* relaxometry by nonlocal means. Magn Reson Med. 2014; 72:260-8.

182. Sandino CM, Kellman P, Arai AE, Hansen MS, Xue H. Myocardial T2* mapping: influence of noise on accuracy and precision. J Cardiovasc Magn Reson. 2015;17:7

183. Feng $Y$, He T, Gatehouse PD, Li X, Harith Alam M, Pennell DJ, et al. Improved MRI R2 * relaxometry of iron-loaded liver with noise correction. Magn Reson Med. 2013;70:1765-74

184. Baeßler B, Schaarschmidt F, Dick A, Stehning C, Schnackenburg B, Michels G, et al. Mapping tissue inhomogeneity in acute myocarditis: a novel analytical approach to quantitative myocardial edema imaging by T2-mapping. J Cardiovasc Magn Reson. 2015;17:115.

185. Lustig M, Donoho D, Pauly JM. Sparse MRI: The application of compressed sensing for rapid MR imaging. Magn Reson Med. 2007:58:1182-95.

186. Feng L, Otazo R, Jung H, Jensen JH, Ye JC, Sodickson DK, et al. Accelerated cardiac T2 mapping using breath-hold multiecho fast spin-echo pulse sequence with k-t FOCUSS. Magn Reson Med. 2011;65:1661-9.

187. Clique H, Cheng H-LM, Marie P-Y, Felblinger J, Beaumont M. 3D myocardial T1 mapping at 3T using variable flip angle method: pilot study. Magn Reson Med. 2014;71:823-9.

188. McDiarmid AK, Swoboda PP, Erhayiem B, Lancaster RE, Lyall GK, Broadbent DA, et al. Athletic cardiac adaptation in males is a consequence of elevated myocyte mass. Circ Cardiovasc Imaging. 2016;9:e003579.

189. Kvernby S, Warntjes MJB, Haraldsson H, Carlhäll C-J, Engvall J, Ebbers T. Simultaneous three-dimensional myocardial T1 and T2 mapping in one breath hold with 3D-QALAS. J Cardiovasc Magn Reson. 2014;16:102.

190. Santini F, Kawel-Boehm N, Greiser A, Bremerich J, Bieri O. Simultaneous T1 and $\mathrm{T} 2$ quantification of the myocardium using cardiac balanced-SSFP inversion recovery with interleaved sampling acquisition (CABIRIA). Magn Reson Med. 2015;74:365-71.
191. Hamilton Jl, Jiang Y, Chen Y, Ma D, Lo W-C, Griswold M, et al. MR fingerprinting for rapid quantification of myocardial T1, T2, and proton spin density. Magn Reson Med. 2017;77(4):1446-458.

192. Sullivan DC, Obuchowski NA, Kessler LG, Raunig DL, Gatsonis C, Huang EP et al. Metrology standards for quantitative imaging biomarkers. Radiology. 2015;277:813-25.

193. Haaf P, Garg P, Messroghli DR, Broadbent DA, Greenwood JP, Plein S. Cardiac T1 Mapping and Extracellular Volume (ECV) in clinical practice: a comprehensive review. J Cardiovasc Magn Reson. 2016;18:89.

194. Captur G, Manisty C, Moon JC. Cardiac MRI evaluation of myocardial disease. Heart. 2016:102:1429-35.

\section{Submit your next manuscript to BioMed Central and we will help you at every step:}

- We accept pre-submission inquiries

- Our selector tool helps you to find the most relevant journal

- We provide round the clock customer support

- Convenient online submission

- Thorough peer review

- Inclusion in PubMed and all major indexing services

- Maximum visibility for your research

Submit your manuscript at www.biomedcentral.com/submit
Biomed Central 